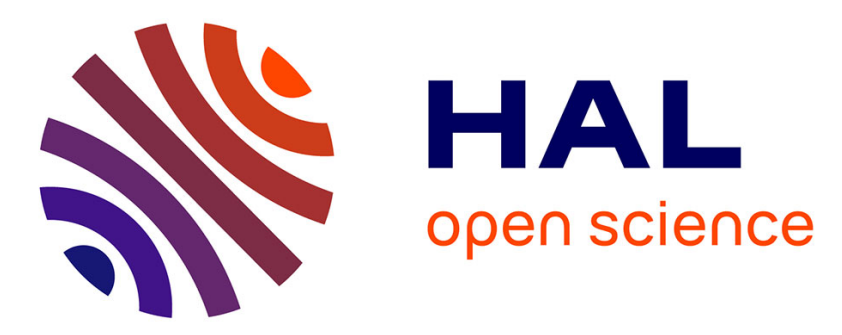

\title{
Investigation of flow features around shallow round cavities subject to subsonic grazing flow
}

\author{
Olivier Marsden, Christophe Bogey, Christophe Bailly
}

\section{To cite this version:}

Olivier Marsden, Christophe Bogey, Christophe Bailly. Investigation of flow features around shallow round cavities subject to subsonic grazing flow. Physics of Fluids, 2012, 24, pp.125107. 10.1063/1.4772194. hal-00780221

\section{HAL Id: hal-00780221 \\ https://hal.science/hal-00780221}

Submitted on 8 Apr 2016

HAL is a multi-disciplinary open access archive for the deposit and dissemination of scientific research documents, whether they are published or not. The documents may come from teaching and research institutions in France or abroad, or from public or private research centers.
L'archive ouverte pluridisciplinaire $\mathbf{H A L}$, est destinée au dépôt et à la diffusion de documents scientifiques de niveau recherche, publiés ou non, émanant des établissements d'enseignement et de recherche français ou étrangers, des laboratoires publics ou privés. 


\title{
Investigation of flow features around shallow round cavities subject to subsonic grazing flow
}

\author{
Olivier Marsden, ${ }^{\text {a) }}$ Christophe Bogey, and Christophe Bailly \\ Laboratoire de Mécanique des Fluides et d'Acoustique, UMR CNRS 5509, \\ Ecole Centrale de Lyon, Université de Lyon, 69134 Ecully Cedex, France
}

(Received 7 February 2012; accepted 9 November 2012; published online 27 December 2012)

\begin{abstract}
The focus of this work is the study of the asymmetric mean flow which can appear inside shallow cavities of circular planform, and for which limited experimental data is available. To this end, the behaviour of cylindrical cavities in grazing flow is investigated numerically, for varying ratios of diameter to depth. Large-eddy simulations (LESs) of cylindrical cavities of diameter $D=10 \mathrm{~cm}$ and depths $H$ ranging from 1 to $10 \mathrm{~cm}$, grazed by a flow at a Mach number 0.25 , are reported. The diameter-based Reynolds number of these cavity flows is 600000 . The incoming boundary layer, of thickness $\delta / D=0.17$, is numerically tripped, in order to reach a highly disturbed state upstream of the cavity. Numerical flow results are compared to experimental findings obtained for similar configurations, and mean flow dependence on the ratio $H / D$ is shown to be correctly reproduced. For cavities of depth greater than 0.7 times their diameter, a symmetric mean flow is obtained, as expected. For shallower cavities, of depth between 0.4 and 0.7 times the diameter, an asymmetric mean flow regime is observed, as previously described in a few experimental papers. Over a small range of depths, from 0.2 to 0.4 times the diameter, very low frequency flow unsteadiness or switching is found. Based on the LES results, a detailed description of these different flow patterns, in terms of both steady and unsteady aspects, is proposed. This description both confirms and adds to the relatively small amount of experimental data available for shallow cylindrical cavity flows. (c) 2012 American Institute of Physics. [http://dx.doi.org/10.1063/1.4772194]
\end{abstract}

\section{INTRODUCTION}

The development of flow in and around cavities has been the subject of much research since the 1930s and the pioneering work of Friesing. ${ }^{1}$ The additional drag generated by a cutout in a flat surface subject to a grazing boundary layer was of primary interest at the time, in particular to the nascent aeronautical industry. With the advent of experimental techniques allowing the study of non-stationary flow fields, the focus shifted to understanding the nature of the flow generated by cavities. The increased interest in the study of more fundamental aspects of cavity flows led to rectangular cavities being privileged in the numerous experimental campaigns performed since the 1950s. As a consequence, less effort has been devoted to the study of cavities of non-rectangular shapes, including those of circular cross section of interest in the present work.

Nevertheless, a few publications have studied some aspects of the flow around cylindrical cavities, and accordingly a brief summary of existing knowledge is given. The general behaviour of cylindrical cavities subject to grazing flows is highly dependent on the ratio of their depth to diameter, which will henceforth be referred to as $\kappa$, defined by $\kappa=H / D$. Deep cavities, for which $\kappa$ is greater than approximately four, exhibit marked acoustic resonance at discrete frequencies which are the odd multiples of the quarter wavelength depth mode. These frequencies are thus independent of the incoming flow velocity. Panpipes ${ }^{2}$ and to a lesser extent flue organ pipes ${ }^{3}$ are an example of this

\footnotetext{
a)Electronic mail: olivier.marsden@ec-lyon.fr.
} 
type of behaviour. For large values of $\kappa$, the geometry of the mouth opening plays a minimal role in frequency selection, and rectangular cavities exhibit similar acoustic trends. Yang et al. ${ }^{4}$ performed a detailed investigation of deep rectangular cavities, illustrating the effect of $\kappa$, of the Mach number as well as of the ratio of cavity length to boundary layer thickness on observed frequencies and on generated acoustic fluctuation levels. Deep cylindrical cavities have also been studied in the context of hydraulic systems, where severe coupling between flow and acoustic resonances in side branches have been observed. ${ }^{5-9}$

For cylindrical cavities where $\kappa$ is between roughly one and four, strong tonal radiation is again observed, but tone frequencies in this range of depths depend not only on cavity depth but also on flow speed. This was observed for example by Elder, ${ }^{10}$ Parthasarathy, ${ }^{11}$ and more recently by Marsden et al. ${ }^{12}$

For shallower cavities, the smaller depth is no longer sufficient to generate strong acoustic tones. However, the flow inside shallow cavities exhibits interesting features. The first studies were performed by German scientists around the start of the second world war. Friesing, ${ }^{1}$ and a little later Wieghardt, ${ }^{13}$ performed some of the first systematic measurements of the drag associated with surface cut-outs and irregularities. Among the different cut-outs studied, cylindrical cavities were included. Some thirty years later, Gaudet and Winter ${ }^{14}$ revisited the study of drag induced by obstacles in boundary layers. They obtained precise measurements of the drag caused by various holes and protuberances placed in a flat plate boundary layer over a wide range of Mach numbers. Both the Mach number range and the variety of geometries studied were chosen to be relevant to the aeronautical industry, for subsonic as well as supersonic aircraft. Their work was the first to pinpoint a strong non-monotonic variation of cavity-induced drag with depth in the case of cylindrical cavities. They were also the first to report the existence of an asymmetric mean flow regime for certain cavity depths, observed thanks to oil film visualisation, and they noted that cavity drag reached a maximum for depths exhibiting strong mean flow asymmetry. The most detailed study to date was performed by Hiwada et al., ${ }^{15}$ and will be referred to subsequently as HKMK. They provided wall pressure measurements, both static and fluctuating, as well as the local wall heat transfer coefficient, for cavity depth ratios between $0.2 \leq \kappa \leq 1$. Their static pressure measurements showed the existence of two asymmetric mean flow regimes. The first is bistable, with two stable states antisymmetrical with respect to the $(x, z)$ plane, see Figure 1 . The flow can be switched from one state to the other by perturbing the free stream conditions upstream of the cavity, as noted by HKMK. This regime was observed for cavities of depth ratio in the range $0.4 \leq \kappa \leq 0.6$. In the second regime, the cavity flow hops from one asymmetrical state to the other by itself, apparently at random, albeit long, intervals. HKMK deduced this regime from pressure recordings on the cavity

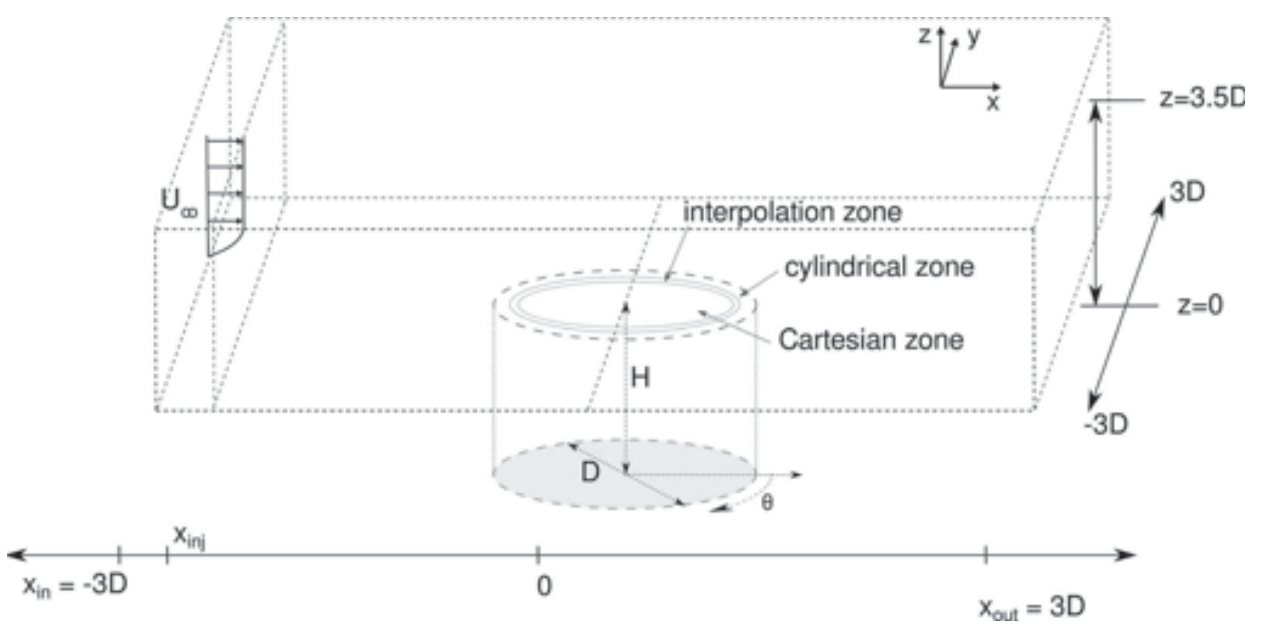

FIG. 1. Sketch (not to scale) of the computational domain. 
wall, and referred to this behaviour as flapping. The range of depths for which flapping was observed was $0.2 \leq \kappa<0.4$. More recently, Dybenko and Savory ${ }^{16}$ performed hot-wire measurements in addition to static and fluctuating pressure measurements for configurations similar to those studied by HKMK. They showed that the flow asymmetry found inside the cavity for certain depths extends well into the cavity's wake. They also proposed that high levels of drag found for certain cavity depths might be linked to strong shear layer oscillations in these cases. Dybenko also provided a succinct experimental confirmation ${ }^{17}$ of the flapping regime first observed by HKMK. Haigermoser et al. ${ }^{18}$ used stereoscopic and tomographic PIV (particle image velocimetry) to examine a cavity of $\kappa=0.5$. Their results highlight the skewed nature of the asymmetrical mean flow found for this configuration. Finally Marsden et al. ${ }^{12}$ characterized the flow and acoustic field generated by cylindrical cavities of depth close to their diameter, and described the strong tonal depth-dependent whistling observed at certain velocities. In this work, unsteady numerical simulations are used to study flow features in cylindrical cavities for $\kappa$ varying between 0.1 and 1.0 with $D=10 \mathrm{~cm}$ in all cases, at a diameter based Reynolds number of $\operatorname{Re}_{D}=U_{\infty} D / v=600000$ and a ratio of diameter to boundary layer thickness of $D / \delta=5.9$, at a Mach number of $M=0.25$. Boundary layer thickness throughout this work is taken as the $99 \%$ free-stream velocity thickness. The diameter and boundary layer thickness have been chosen to allow the deepest cavity studied here, of depth ratio $\kappa=1$, to be compared to detailed experimental data ${ }^{19}$ obtained under similar conditions. The different flow regimes found over the range $0.1 \leq \kappa \leq 1$ are studied in detail. Four distinct flow regimes are identified, as postulated by HKMK. Mean and fluctuating quantities are used to illustrate various aspects of these flow fields. Particular attention is payed to the flapping phenomenon identified by HKMK for $0.2 \leq \kappa \leq 0.4$. Drag values obtained from the computations are compared to values found in the literature. The origin of the increased drag found for certain cavity depths is discussed.

\section{NUMERICAL PROCEDURE AND PARAMETERS}

\section{A. Numerical procedure}

The simulations are performed by solving the filtered compressible three-dimensional NavierStokes equations. The equations are formulated on conservative variables, namely, density $\rho$, momentum $\rho(u, v, w)$ and total energy $\rho e_{T}$. Air is modeled as a perfect gas at standard atmospheric conditions, and molecular dynamic viscosity is computed via Sutherland's law. These equations are presented in detail in Bogey and Bailly. ${ }^{20}$ Spatial derivatives are computed using low-dispersion and low-dissipation finite-difference schemes. ${ }^{21}$ For the computation of spatial derivatives away from boundaries, centred fourth-order eleven-point finite differences are used, while close to boundaries, be they solid walls or far field conditions, non-centred eleven-point schemes are implemented. ${ }^{22}$ The second derivatives contained in the viscous stress terms are computed by two successive applications of the finite differences. Time integration is performed with an explicit second-order six-stage Runge-Kutta method. ${ }^{21}$ A CFL condition with a value of 1 based on the smallest grid dimension is used to set the time step. The large-eddy simulation (LES) approach followed in this work, referred to as relaxation filtering or LES-RF, is based on the explicit application of a low-pass optimized 11-point filter ${ }^{21}$ operation to the flow variables, in order to take into account the dissipative effects of the subgrid scales by relaxing turbulent energy only through the smaller discretized scales. This can be considered a functional dissipation model, as opposed to the structural models found in the literature. ${ }^{23}$ The LES-RF approach has been successfully implemented in previous simulations of subsonic round jets, ${ }^{24,25}$ airfoils ${ }^{26}$ and round cavities. ${ }^{19}$ A detailed discussion of this technique can be found in the work of Bogey and Bailly. ${ }^{20}$ In order to allow the complex geometry to be treated with high order methods, an overset multi-block domain is constructed, with cylindrical coordinates being used to discretize the circular cavity wall, and Cartesian coordinates for both the centre of the cavity and the outer flow zone. In the overset grid zones, inter-grid communication is carried out via interpolation methods optimized in the wavenumber space. ${ }^{27}$ Centred ten-point interpolation stencils are used wherever possible. Close to solid walls, it is not always possible to perform centred optimization due to a lack of donor points, and in such cases stable non-centred optimized interpolation schemes are deployed. ${ }^{28}$ Code parallelisation is based on MPI, and each 
TABLE I. Number of grid points, in millions, in the cavity zone of the reported computations.

\begin{tabular}{|c|c|c|c|c|c|c|c|c|c|c|c|c|c|c|}
\hline$\kappa$ & 0.1 & 0.15 & 0.2 & 0.3 & 0.32 & 0.33 & 0.35 & 0.4 & 0.45 & 0.5 & 0.6 & 0.7 & 0.8 & 1.0 \\
\hline M points & 1 & 1.6 & 2 & 2.7 & 2.8 & 2.8 & 2.9 & 3 & 3 & 3.1 & 3.4 & 3.7 & 4 & 4.5 \\
\hline
\end{tabular}

computation is split into forty six blocks. Care is taken to balance the distribution of computational cost among cores, allowing, for example, a sustained aggregate performance of $40 \mathrm{GFlop} / \mathrm{s}$ to be reached on 46 Intel Xeon 5540 cores.

\section{B. Parameters}

For all flow configurations, the free stream flow velocity is $90 \mathrm{~m} \mathrm{~s}^{-1}$, and a boundary layer thickness of $16 \mathrm{~mm}$ is chosen, to match the upstream experimental conditions from Marsden et al. ${ }^{12,19}$ A turbulent mean profile of thickness $\delta=0.17 \mathrm{D}$, based on a single equation model, ${ }^{29,30}$ is imposed at the entry 3 diameters upstream of the cavity, and slightly downstream of the entry plane volumetric force terms are injected in order to generate turbulent-like fluctuations in the boundary layer before it reaches the cavity. The injection process and the resulting boundary layer characteristics are described in the Appendix. The Reynolds number based on the upstream momentum thickness $\delta_{\theta}$ is $\operatorname{Re}_{\theta}=4000$. For all cavity depths, the computations are run for a physical duration of at least $1 \mathrm{~s}$, corresponding to 700000 time steps and nine hundred free stream convective times across the cavity opening.

A total of fourteen computations with different values of $\kappa$ have been performed, over the range $\kappa \in[0.1,1]$, see Table I. Figure 1 provides a sketch of the computational domain, whose description is given below. The outer computational domain, excluding the exit sponge zone, covers the range $-3<x / D<3,-3<y / D<3$, and $0<z / D<3.5$, and is discretized by $495 \times 350 \times 128$ points in the streamwise $x$, cross-stream $y$, and vertical $z$ directions, respectively. The number of grid points inside the cavity is reported in Table I for each depth. Grid spacings relative to the boundary layer thickness $\delta$ and to those at the cavity mouth are shown in Figure 2 for the $\kappa=0.2$ case. For all depths, grid spacings at the cavity mouth for the Cartesian mesh are given by $\Delta x / \delta=\Delta y / \delta=4.3$ $\times 10^{-2}$ and $\Delta z / \delta=1.8 \times 10^{-2}$. At the cavity wall, the radial spacing of the cylindrical grid is given by $\Delta r / \delta=1.8 \times 10^{-2}$, and its azimuthal spacing by $R \Delta \theta / \delta=6 \times 10^{-2}$. Vertical spacing along the cavity wall is the same as that in the Cartesian central part of the cavity. The outer grid is stretched in every direction away from the cavity, at a maximum geometrical rate of 1.04.

For the cavity of depth ratio $\kappa=1.0$, comparisons are made with experimental results from Marsden et al. ${ }^{12,19}$ For the shallower cavities, experimental flow results are available for certain depths in the work of Gaudet and $\operatorname{Winter}^{14}(\kappa \leq 0.3, M=0.2, \delta / D=1.25)$, Hiwada et al. ${ }^{15}$ $\left(0.1 \leq \kappa \leq 1.0, M=0.075, \delta / H=0.4, \operatorname{Re}_{D}=10^{4}\right)$, Dybenko et al. ${ }^{31}(\kappa=0.2,0.47,0.7$, $\left.M=0.08, \operatorname{Re}_{D}=1.3 \times 10^{5}, \delta / D=0.72\right)$ and Haigermoser et al. ${ }^{18}(\kappa=0.5, M=0.036$,
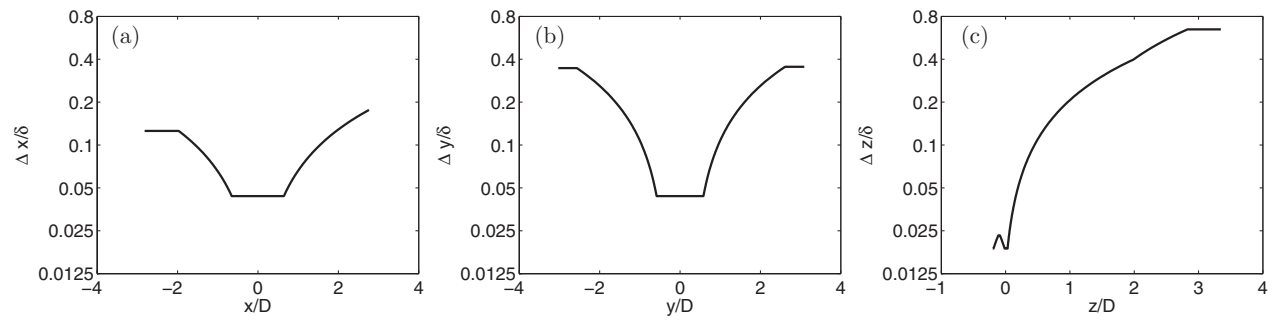

FIG. 2. Grid spacings in the (a) streamwise $(\Delta x)$, (b) cross-stream $(\Delta y)$, and (c) vertical ( $\Delta z$ ) directions, non-dimensionalized by the boundary layer thickness $\delta$. Cavity of depth ratio $\kappa=0.2$. 
$\delta / D=0.5625)$. Comparisons are made between the present numerical results and data from these studies wherever possible.

\section{RESULTS}

\section{A. Baseline cavity of depth $\kappa=1$}

There is little quantitative data available for shallow cylindrical cavities to which the present computational results can be compared. As a consequence, although not exhibiting the asymmetrical features of particular interest in this work, the $\kappa=1$ cavity flow is compared to experimental data from Marsden et al. ${ }^{19}$ established for the same cavity geometry at a slightly lower Mach number of 0.21 .

Figures 3(a) and 3(b) show profiles of mean streamwise velocity and of rms streamwise fluctuations, respectively. The mean streamwise velocity is normalized by the free stream velocity, while velocity fluctuation levels are normalized by the maximum turbulence level measured in the boundary layer upstream of the cavity, of $15 \%$ both numerically and in the experiment. ${ }^{19}$ Experimental data is represented by the circles, while computational data is represented by solid lines. Despite the difference in Mach number between the two configurations, good agreement is observed, both in terms of mean velocity and in terms of streamwise fluctuations. A large confined vortex can be seen in the mean streamwise velocity plot, similar to those found in shear-driven square cavities. ${ }^{32,33}$ The recirculation is intense, with a maximum time-averaged downwards velocity along the downstream wall of $30 \%$ of the free stream velocity, and a maximum velocity in the upstream direction along the cavity floor of $26 \%$ of $U_{\infty}$. In the velocity fluctuation plot, a rapid transition from a boundary layer profile to a roughly antisymmetrical shear layer profile can be noted. Inside the cavity, fluctuation levels close to the cavity floor are very large. Indeed, the ratio of rms velocity fluctuations to local mean velocity reaches values of around $25 \%$.

The three-dimensional nature of the mean flow inside the cavity can be ascertained from the static wall pressure, illustrated in Figure 4 where the pressure coefficient $C_{p}=\left(p-p_{\infty}\right) /\left(\rho_{\infty} U_{\infty}^{2} / 2\right)$ is represented on the cavity wall and floor. The experimental data and results from the computation are in good qualitative agreement. A symmetrical static pressure field is observed, indicating that the mean flow inside the cavity is also symmetrical with respect to the $(x, z)$ plane. A large area of high pressure is located at the downstream edge of the cavity lip around $-0.2<z / H<0$ and $-50^{\circ}$ $<\theta<50^{\circ}$. This zone is generated by the impact of the shear layer on the cavity wall, and reaches a maximum value of $C_{p}=0.18$ in the computation, which is less intense than values observed for rectangular cavities of similar dimensions and at similar flow speeds, ${ }^{32,34}$ although notably higher than the values observed experimentally. Two zones of negative $C_{p}$, centred around $\theta= \pm 90^{\circ}$ and $z / H=-0.45$, confirm the presence of a large central captive vortex inside the cavity. On the cavity

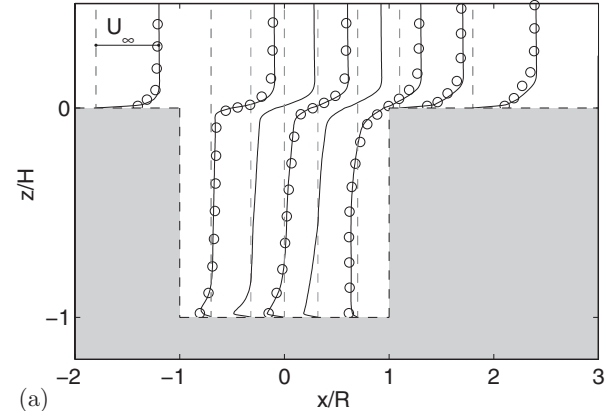

FIG. 3. Profiles of (a) mean streamwise velocity and (b) rms values of streamwise velocity fluctuations for a cylindrical cavity of depth ratio $\kappa=1.0$ and diameter $D=2 R=10 \mathrm{~cm}$. Computational data at $M=0.25$ (一); experimental data at $M$ $=0.21(000)$.

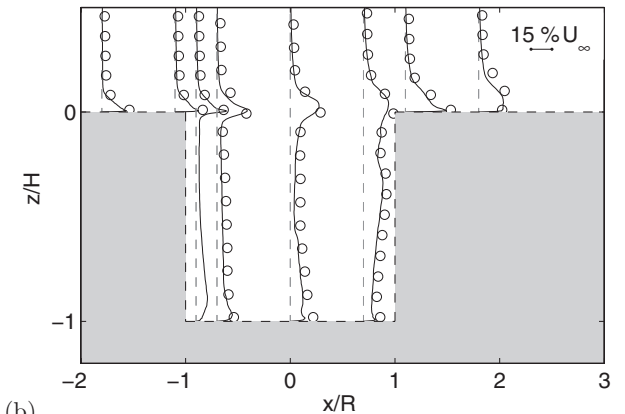

(b) 


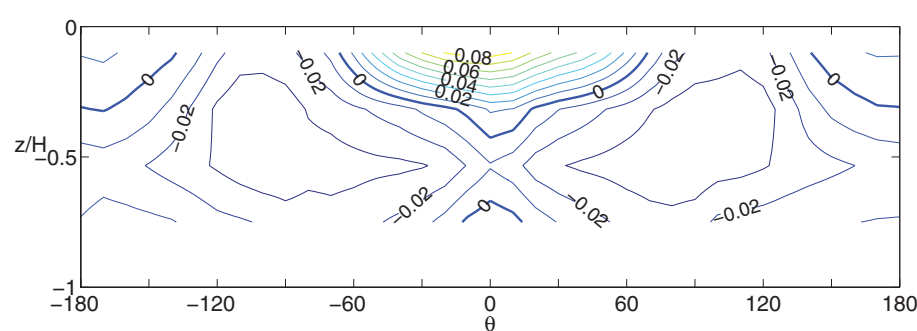

(a)

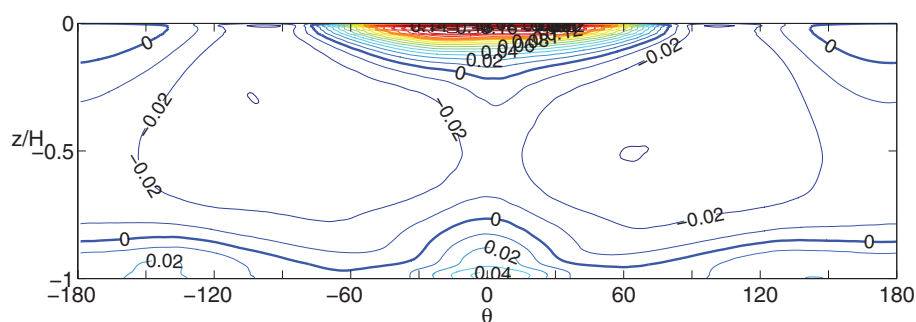

(c)

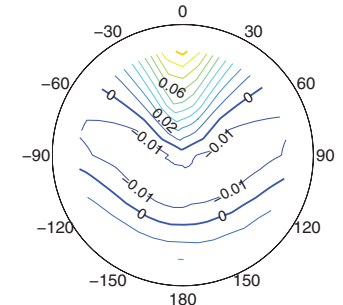

(b)

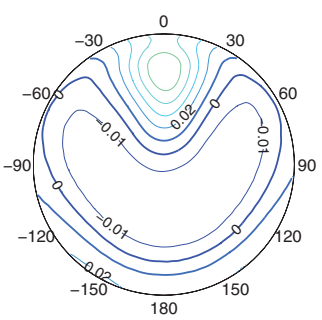

(d)

FIG. 4. Wall pressure coefficient $C_{p}$ ((a) and (c)) on the cavity wall and ((b) and (d)) cavity floor for the case $\kappa=1$. Experimental data at $M=0.21$ (top), and computational data at $M=0.25$ (bottom). Isopleth scale between -0.03 and 0.15 .

floor, isopleths are curved in a characteristic horseshoe shape, indicating that the central vortex is also curved in the cross stream $y$ direction. The impact of the main recirculation on the floor is clearly visible with the strong positive value of $C_{p}=0.06$ centred around $\theta=0$ and $r / R=0.7$. Computational results on the cavity floor suggest the presence of two counter-rotating vortices at the bottom of the cavity, a narrow one along the downstream edge stretching roughly $30^{\circ}$ to either side of the $\theta=0$ downstream direction, and a wider one along the upstream edge, spanning $\pm 90^{\circ}$ around the $\theta=180^{\circ}$ upstream direction.

The computational data allows the structure of the mean flow field to be examined in more detail. Profiles of the cross stream $V$ mean flow inside the cavity, as well as a vector plot of the $(V, W)$ field, in the $x=0$ plane, are shown in Figure 5(a). Profiles of $V$ are normalized by the maximum recorded value of the cross stream mean flow, $\max (V)$, and indicated in black dashed lines, while

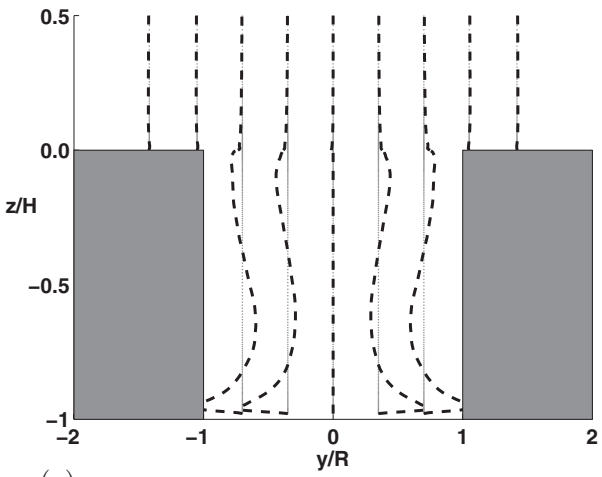

(a)

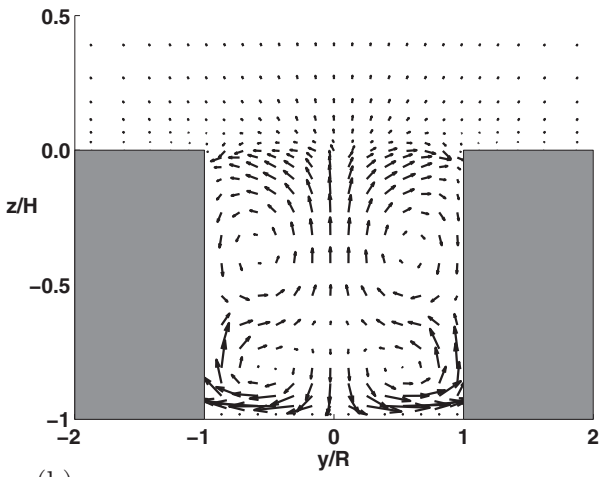

(b)

FIG. 5. (a) Normalized profiles of mean cross-stream $V$ velocity in dashed lines, and (b) normalized vector plot of $(V, W)$ simulation data for the cavity of $\kappa=1$. 


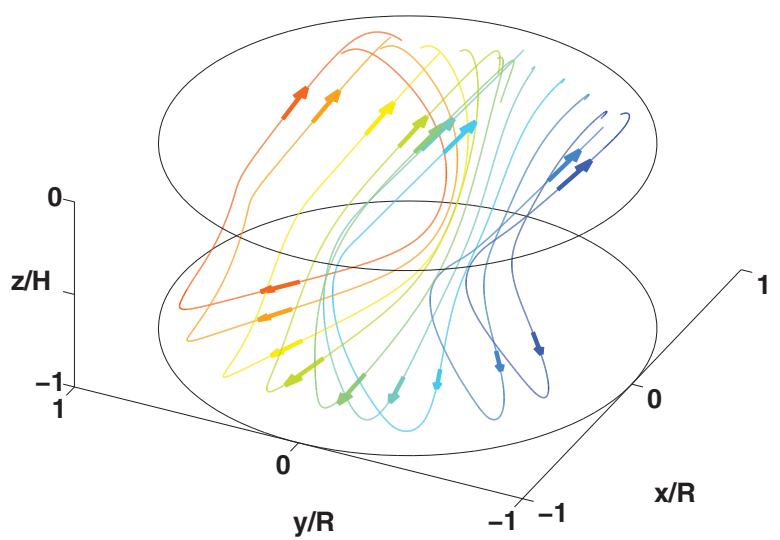

FIG. 6. Streamlines computed from the mean flow field inside a cavity of depth ratio $\kappa=1$.

the vector plot is normalized by the maximum in-plane velocity, $\max \left(\sqrt{V^{2}+W^{2}}\right)$, and represented in gray. The cross stream velocity profiles show that in the $x=0$ plane, i.e., halfway along the cavity in the flow direction, close to the cavity floor and to a lesser extent just under the shear layer, fluid is heading away from the plane of symmetry, while halfway up the cavity, fluid converges towards this plane. This is also clearly visible in the vector plot of $V$ and $W$, shown in Figure 5(b), where two sets of counter-rotating vortical structures appear, the upper pair centred around $z / H=-0.4$, and the lower pair around a height of $z / H=-0.85$.

Figure 6 attempts to illustrate the general shape of the main captive vortex inside the cavity by representing streamlines computed from the mean flow field. The streamline seed points are located on the arc $r / R=0.42$ at a height of $z / H=-0.05$, and consecutive points are separated by an angle of $\pi / 16$. The corresponding streamlines are coloured according to the cross stream $y$ coordinate of the seed points, cold colours corresponding to negative values of $y$ and warm colours to positive values. Arrows on the streamlines indicate local mean flow direction. The large central recirculation is clearly identified by the streamlines, confirming the previously described similarity with mean flow in a square cavity. However, the recirculation's shape is strongly affected by the cylindrical wall, as demonstrated by the large $V$ component giving the streamlines their highly 3D aspect. Streamlines, shown in Figure 6, are seen to converge during their downwards movement along the downstream wall, and then diverge strongly as they head upstream along the cavity floor before finally reconverging slightly as they resascend the upstream cavity wall.

\section{B. Flow variation as depth is decreased}

\section{General trends}

It has been known since the work of Gaudet and Winter ${ }^{14}$ that shallow round cavities can produce asymmetrical mean flows. HKMK categorized these cavities into four depth-to-diameter ranges based on the resulting flow regime, and provided limited experimental data for certain aspects of these four regimes. Other than their work, there remains, however, little quantitative data available on such flows. The four flow regimes proposed by Hiwada et al. ${ }^{15}$ are reproduced numerically, and descriptions of each regime are given in what follows.

An overview of the effect of the depth to diameter ratio $\kappa$ on the mean flow is given in Figures $7(\mathrm{a})-7(\mathrm{j})$ by representing static pressure fields, in non-dimensional $C_{p}$ form, on the cavity walls and in Figures $8(a)-8(j)$ on the cavity floor, for a selection of cavity depth ratios. For the first three depths, $\kappa=1,0.8$, and 0.7 , the pressure fields on both the cavity wall and floor are essentially 
(a)

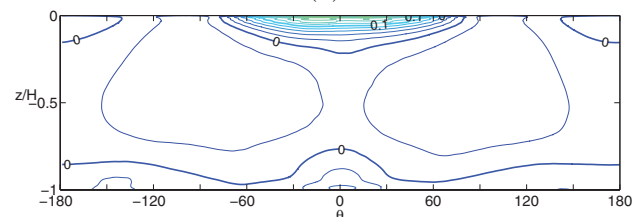

(b)

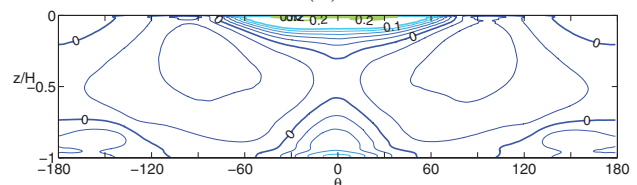

(c)

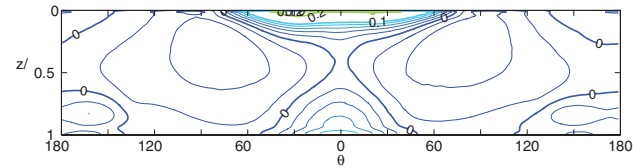

(d)

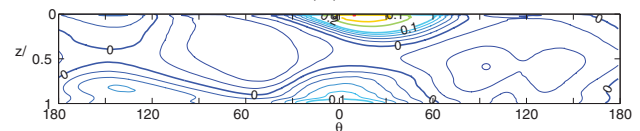

(e)

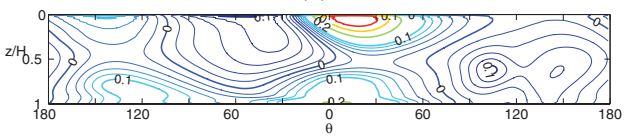

(f)

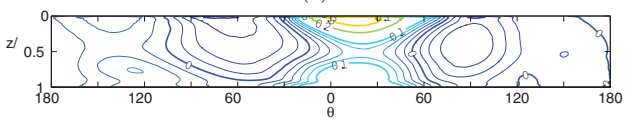

(g)

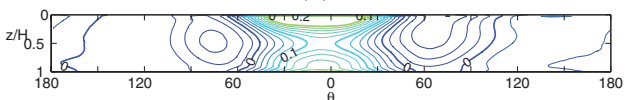

(h)

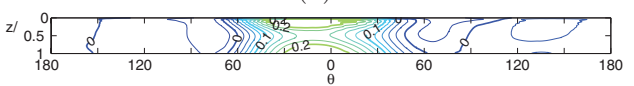

(i)

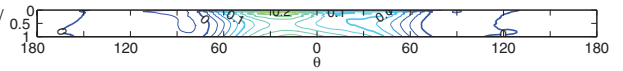

(j)

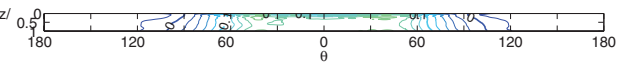

FIG. 7. Static pressure coefficient $\left(C_{p}\right)$ distribution on the cavity wall for $0.1 \leq \kappa \leq 1.0$, (a) $\kappa=1$, (b) $\kappa=0.8$, (c) $\kappa=0.7$, (d) $\kappa=0.6$, (e) $\kappa=0.5$, (f) $\kappa=0.4$, (g) $\kappa=0.32$, (h) $\kappa=0.2$, (i) $\kappa=0.15$, and (j) $\kappa=0.1$.

symmetrical, with slight deviations visible in the case of $\kappa=0.7$. The two characteristic low pressure zones located around $90^{\circ}$ to either side of the free stream flow direction, and attributed to the central captive vortex flow inside the cavity, are seen to increase in intensity as cavity depth decreases, suggesting a progressive strengthening of the recirculation. The following three depths, $\kappa=0.6,0.5$, and 0.4 , all exhibit highly asymmetrical mean pressure fields. The aforementioned zones of negative pressure coefficient are seen to rotate together around the cavity's axis, and to change altitudes, one of the two zones rising towards the cavity opening and the other dropping towards the floor. Static pressure asymmetry is most marked for a depth ratio of 0.5. It is also for this value of $\kappa$ that the static pressure at the shear layer impact point, visible in Figure 7(e) at around $\theta=20^{\circ}$, reaches its maximum.

Pressure fields associated with cavities of depth smaller than $\kappa=0.32$, shown in Figures 7 and 8 from ( $g$ ) to $(\mathrm{j})$, are seen to return progressively to a symmetrical state. The strong pockets of negative pressure observed for deeper cavities are not present in these configurations.

One of the most striking features of shallow cylindrical cavity flows is the appearance of the aforementioned flow asymmetry for a range of depth ratios. A simple means of illustrating the mean flow asymmetry is to examine the deviation of the mean flow along the cavity floor from the symmetrical state. An estimate of this mean field skew is represented in Figure 9, as a function of the cavity depth ratio $\kappa$. This estimate $\Psi$ is defined here by computing the average deviation angle of the mean flow with respect to the $x$ direction, just above the cavity floor, over a disk of radius $D / 4$. This angle is computed as the arithmetic average of the deviation angle at each grid point inside the disk. Dashed vertical lines indicate approximate separations between the different flow regimes described by HKMK, which will be discussed in more detail subsequently. Rotation of the mean flow is seen to appear progressively for depth ratios smaller than $\kappa=0.7$, reaching a maximum of around $35^{\circ}$ for depths around $\kappa=0.5$, before diminishing again and almost disappearing for $\kappa=0.35$. 
(a)

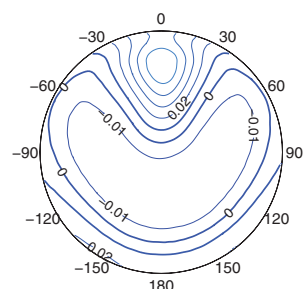

(e)

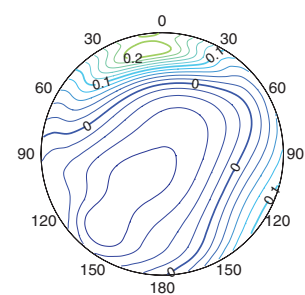

(i)

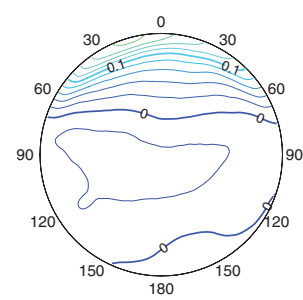

(b)

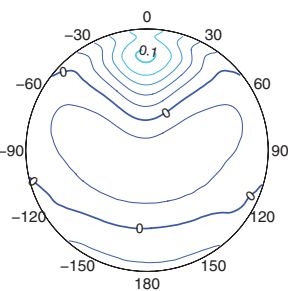

(f)

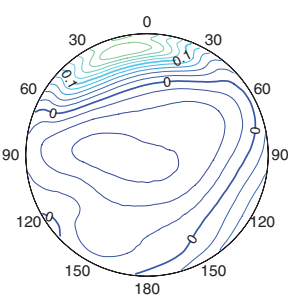

(j)

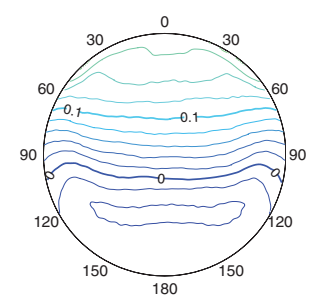

(c)

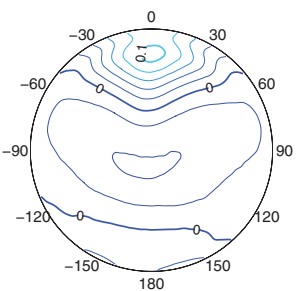

(g)

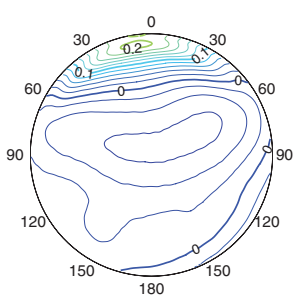

(d)

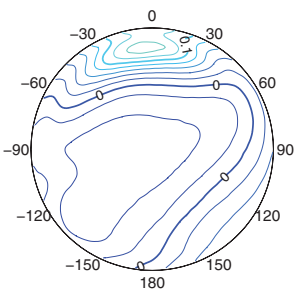

(h)

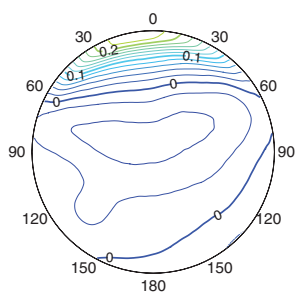

FIG. 8. Pressure coefficient $C_{p}$ on the cavity floor for same depths (see Figure 7 caption).

\section{Depth ratio $0.4<\kappa<0.7$}

The mean flow is now described in detail for a cavity depth of $\kappa=0.5$, and compared to experimental results from HKMK. The tendencies described in what follows are observed to varying degrees for cavity depths in the range $0.4<\kappa<0.7$.

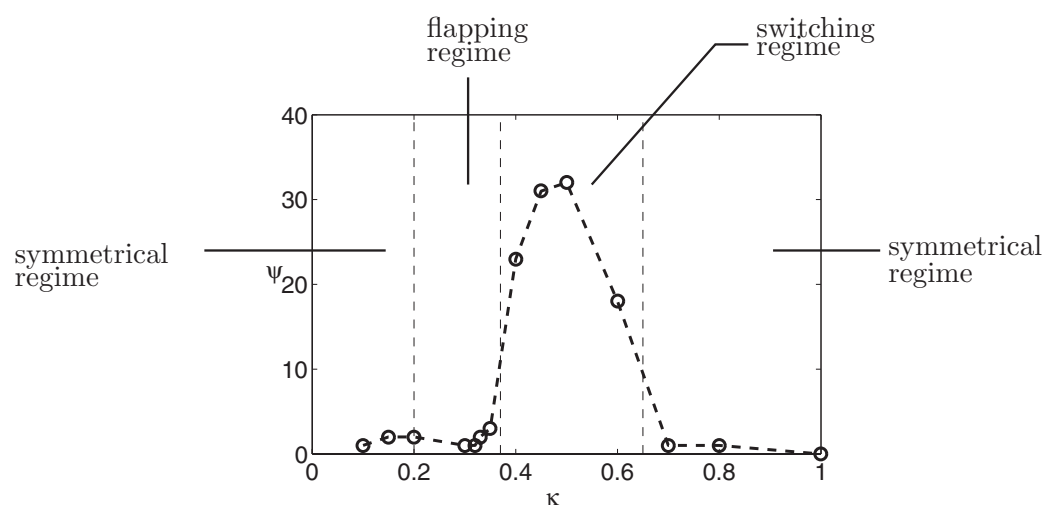

FIG. 9. Mean flow rotation $\Psi$ in degrees around the $z$ axis as a function of cavity depth ratio $\kappa=H / D$. Dashed vertical lines denote separations between different regimes as proposed by HKMK, ${ }^{15}$ and symbols, reported computations. 
(a)

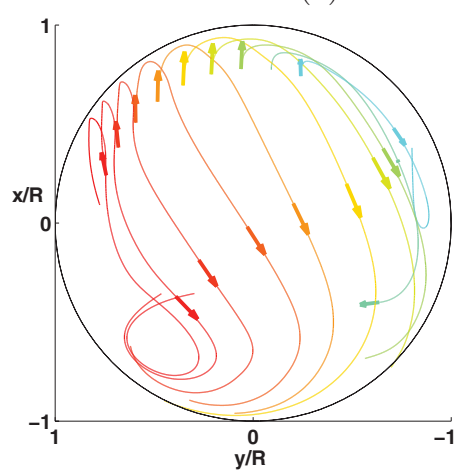

(b)

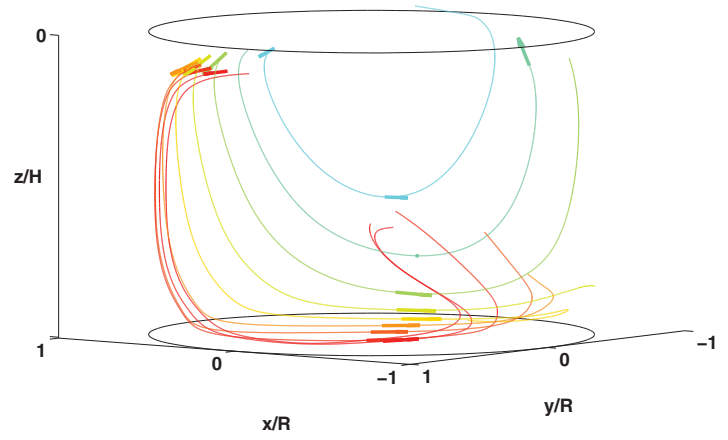

FIG. 10. (a) Top view of streamlines computed from the mean flow field inside the cavity, and (b) side view of streamlines, rotated by an angle of $35^{\circ}$ around the $z$ axis, for a cavity of depth ratio $\kappa=0.5$.

For a depth ratio of $\kappa=0.5$, five successive computations were started from an identical symmetrical initialisation, forced with identical time series of inflow forcing, and allowed to run to a converged mean flow field. Of these five computations, the mean fields of three converged to one side, while those of the other two converged to the second side. This suggests that there is no systematic numerical bias present in the computations, and that imperfections due, for example, to numerical round-off are sufficient to trigger the choice of side to which a given computation will converge. The asymmetrical flow can be considered converged after approximately 50 free stream convection times across the cavity opening. A comparison of flow fields obtained from the two families of mean flows, not presented here, shows as expected a high degree of anti-symmetricity.

Figure 10(a) illustrates the rotation of the mean flow inside the cavity with respect to the exterior flow. It shows streamlines for a cavity of depth $\kappa=0.5$, coloured as in Figure 6 by the cross stream $y$ coordinates of the seed points, viewed from above the cavity. The free stream flow is directed along the $x$ coordinate. The rotation of the cavity flow with respect to the outer flow is clearly visible in the orientation of the streamlines along the cavity floor, as well as in Figure 10(b) which shows the same streamlines from a viewing direction rotated by $35^{\circ}$ with respect to the cross stream $y$ direction. This rotation corresponds well to the value of $\Psi$ given in Figure 9 for $\kappa=0.5$. These streamlines indicate that the rotated flow is fairly homogeneous over a large part of the cavity floor, unlike that of the cavity of depth ratio one, whose streamlines shown in Figure 6 highlight a fan-like spreading of the mean flow as it heads upstream along the cavity floor. The rotation of the mean flow, as well as static pressure on the cavity walls for a depth of $\kappa=0.5$, can be compared to the data published by HKMK for a cavity of identical depth, at a lower diameter-based Reynolds number of $1.1 \times 10^{5}$ and lower flow velocity of $25 \mathrm{~m} \mathrm{~s}^{-1}$. They provided both measurements of static pressure and oil film visualisations on the cavity walls. The oil streaks on the cavity floor in their work point to a rotation of the recirculation by slightly more than $30^{\circ}$, as do the pressure isopleths on the cavity floor. From their oil film visualisations, Gaudet and Winter ${ }^{14}$ also observed a similar rotation of the mean flow for a cavity of depth ratio $\kappa=0.47$, and estimated this rotation at around $45^{\circ}$. A subsequent re-examination of their work by Dybenko ${ }^{17}$ resulted in a lower estimation of around $30^{\circ}$.

HKMK's pressure data are reproduced in Figures 11(a) and 11(b) and compared to the present numerical results shown in Figures 11(c) and 11(d) for the same depth. Although the $C_{p}$ extrema are slightly less intense in the computation, a good qualitative match with the experimental pressure field is found, indicating that the general flow rotation estimated from the numerical data is realistic. The pressure distribution is markedly different to that for the cavity of depth $\kappa=1$, shown in Figure 4. It has been both rotated and distorted, and extrema are more intense. The large negative pockets of $C_{p}$, which are symmetrical for $\kappa=1$, are now highly asymmetrical. Both pockets are rotated in the same direction by roughly $40^{\circ}$, one pocket now being centred around $\theta=-60^{\circ}$ and the other centred around $\theta=130^{\circ}$. The pocket that is closer to the downstream $\theta=0$ direction 
(a)

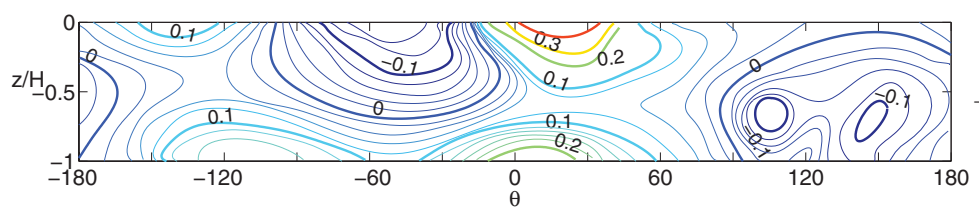

(c)

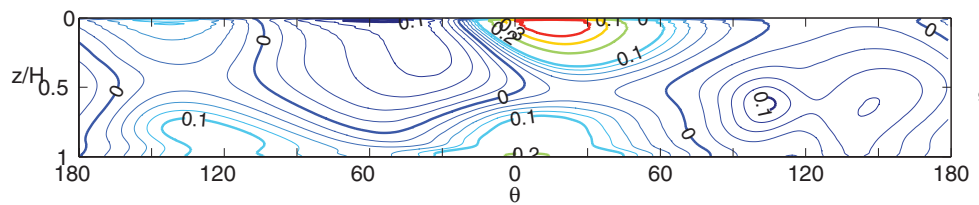

(b)

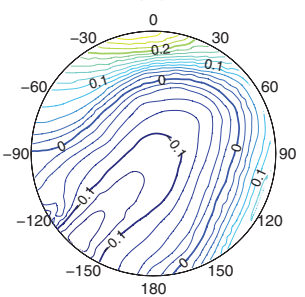

(d)

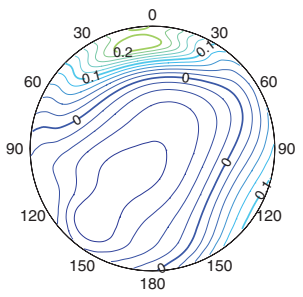

FIG. 11. Static pressure coefficient on the cavity wall ((a) and (c)) and floor ((b) and (d)) for a cavity of depth $\kappa=0.5$. HKMK's data ${ }^{15}$ at $M=0.07$ (top), and present computational data at $M=0.25$ (bottom).

has shrunk, and risen to the cavity opening, while the other has dropped down to $z / H=-0.7$ and increased in size. Both pockets reach negative values lower than 0.1 , and are thus three to four times more intense than those of the $\kappa=1$ cavity. The zone of positive static pressure generated by the shear layer impact on the downstream cavity wall has been shifted by a little under $30^{\circ}$ away from $\theta=0$, and become both smaller and more intense. A maximum value of 0.52 is found in the computation, which is 2.7 times higher than the value of 0.19 obtained for the deep cavity. On the cavity floor, the pressure gradient between the downstream wall and the central zone of the cavity is much stronger than for the deep cavity, and is made up, over a wide zone, of almost parallel contours. This pressure gradient is accompanied by a substantially higher recirculation velocity along the cavity floor, reaching $45 \mathrm{~m} \mathrm{~s}^{-1}$ or half of the free stream velocity, and its parallel aspect mirrors that of the streamlines along the cavity floor shown in Figure 10.

The large static pressure maximum on the cavity side wall can be better understood by examining the streamwise velocity in the $x=0$ plane. This quantity is represented in Figure 12(a), and is strikingly asymmetrical. The shear layer descends into the cavity on the $y>0$ side, and is ejected into the free stream on the $y<0$ side, and it is this warping of the shear layer and its subsequent impact on the downstream wall which generates the strong maximum static pressure around $\theta=15^{\circ}$. Figure 12 (b), showing the two cross stream $V$ and $W$ components of the mean velocity in the $x=0$ plane, illustrates the cause of the shear layer warping, namely the strong rotation around the $x$ axis which pushes the flow up for $y<0$ and sucks it down into the cavity for $y>0$. Mean flow in the cross stream $y$ direction reaches $0.3 \times U_{\infty}$, which is of the same order of magnitude as the mean flow along the cavity floor in the streamwise $x$ direction. This flow structure is very similar to that described by Haigermoser et al. ${ }^{18}$ for a cavity of identical depth ratio, but at a far lower Mach number of 0.036 , and placed under a very thick boundary layer.

The strong recirculation generates high levels of turbulent velocity fluctuations throughout the cavity and in particular in the shear layer. Streamwise and cross stream mean fields and rms fluctuations are shown in Figures 13(a)-13(f) in the streamwise $y=0$ and Figures 14(a)-14(f) for the cross stream $x=0$ planes. The characteristic warped shape of the shear layer already seen in Figure 12(a) is the marking feature of the rms values of the velocity fluctuations in this plane, where levels reach $21 \%$ of the free stream velocity for $u_{r m s}^{\prime}$ and $18 \%$ and $16 \%$ for $v_{r m s}^{\prime}$ and $w_{r m s}^{\prime}$, respectively. A notable pocket of fluctuations is visible in the streamwise plane centred around $x / R$ $=-0.3$ and $z / H=-0.8$, corresponding to the zone where the wall jet formed by the recirculation 

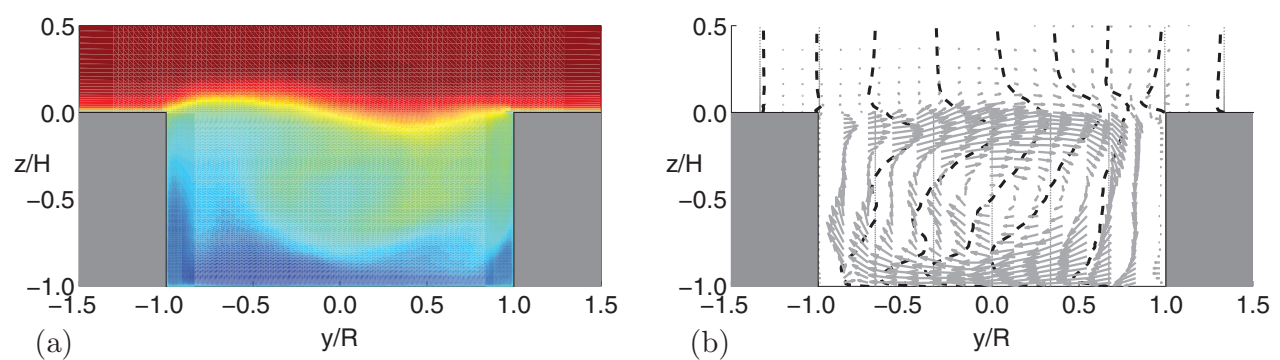

FIG. 12. (a) Computed mean streamwise velocity and (b) cross stream velocity representation in the $x=0$ plane, for a cavity of depth $\kappa=0.5$. Colour scale from -60 to $90 \mathrm{~m} \mathrm{~s}^{-1}$ for streamwise velocity.

along the cavity floor separates from the floor. The large levels of $v_{r m s}^{\prime}$ and $w_{r m s}^{\prime}$ observed here suggest that the wall jet separation is highly unsteady. The levels of velocity fluctuations found in the shear layer are considerably higher than those measured by Haigermoser et al. ${ }^{18}$ but this to be expected given the far larger boundary layer thickness in their work, $\delta / D=0.5625$, than in this study, $\delta / D=0.17$.

\section{Depth ratio $0.2<\kappa<0.4$}

HKMK observed random large scale mean flow oscillations over the range $0.2 \leq \kappa<0.4$, by monitoring static pressure at two symmetrical locations $30^{\circ}$ to either side of the downstream $\theta=0$ direction, just below the cavity opening. They referred to this behaviour as flapping, as opposed to switching for the previously described bi-stable flow regime. The presence of mean flow flapping was recently confirmed experimentally by Dybenko. ${ }^{17}$ One of the striking points of the mean flow flapping found by HKMK was its very large time scale compared to that of the turbulent flow, and this feature is strongly unfavourable for the numerical reproduction of the phenomenon. Indeed, according to HKMK's pressure data for $\kappa=0.37$, value for which the authors found the flapping to be most notable, the characteristic flapping time, referred to subsequently as $T_{f}$, was of the order of 2 to $15 \mathrm{~s}$, or of the order of 800 to 6000 cavity convection times $T_{c}=D / U_{\infty}$, also referred to as fly-over times based on their cavity diameter and flow velocity. The time step dictated by numerical stability constraints in the present simulations is such that 1000 time steps are required for one fly-over period, and thus, assuming that $T_{f} / T_{c}$ is not strongly dependent on Mach

(a)

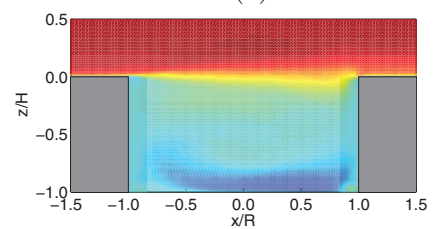

(d)

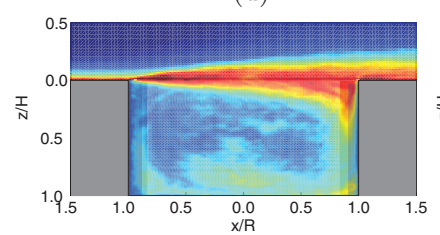

(b)

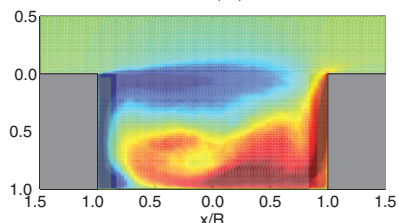

(e)

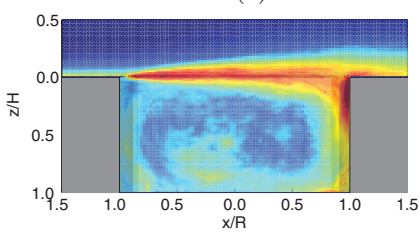

(c)

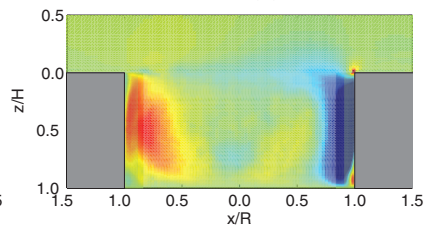

(f)

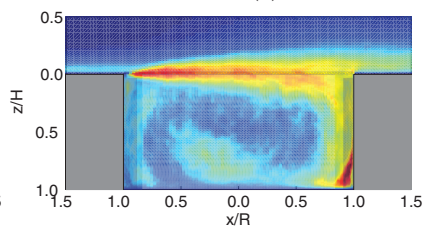

FIG. 13. Mean and rms values of the ((a) and (d)) streamwise $u$, ((b) and (e)) cross stream $v$, and ((c) and (f)) vertical $w$ velocity fields in the $y=0$ plane for a cavity of depth ratio $\kappa=0.5$. Colour scale between -60 and $100 \mathrm{~m} \mathrm{~s}^{-1}$ for $U$ and between -25 and 25 for $V$ and $W$, and between $0 \%$ and $20 \%$ of the free stream velocity for all rms components. 
(a)

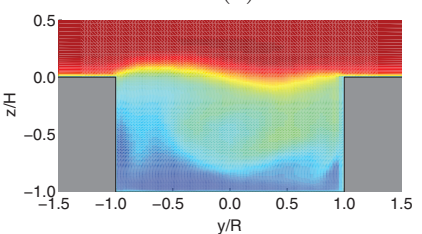

(d)

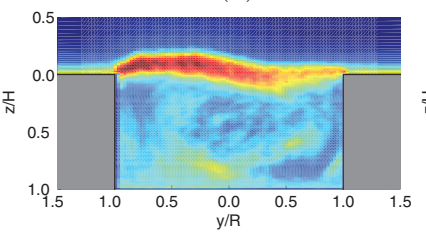

(b)

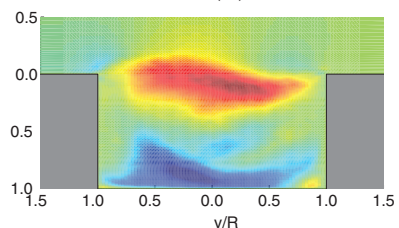

(e)

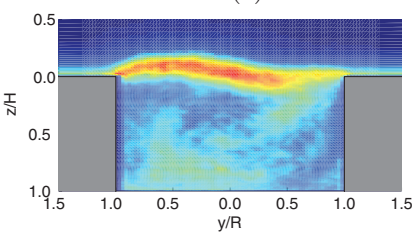

(c)

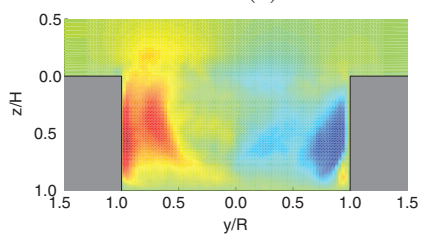

(f)

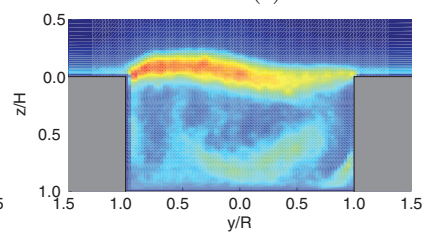

FIG. 14. Mean and rms values of the ((a) and (d)) streamwise $u$, ((b) and (e)) cross stream $v$, and ((c) and (f)) vertical $w$ velocity fields in the $x=0$ plane for a cavity of depth ratio $\kappa=0.5$. Colour scale as in Figure 13.

number or Reynolds number, at least $1 \times 10^{6}$ time steps would be required to reach the order of magnitude of the characteristic flapping time for a cavity of depth ratio $\kappa=0.37$ in the present study. Such a computation was deemed to be unrealistic, and was not attempted.

However, fluctuating pressure signals obtained by HKMK for other depths in the range $0.2 \leq \kappa<0.4$ suggest that the characteristic flapping time increases with $\kappa$, with a value smaller than $1 \mathrm{~s}$ for $\kappa=0.2$, and a value of around 1 to $5 \mathrm{~s}$ for $\kappa=0.3$. Based on the apparent variation of $T_{f}$ with $\kappa$, two computations were performed for smaller depths, in the hope of detecting mean flow flapping within a more manageable number of time steps. Results are presented for cavities of depth ratio $\kappa=0.32$ and $\kappa=0.2$.

Both computations exhibit large-scale very low frequency variations of flow quantities inside the cavity. These swings appear to be similar to the flapping observed experimentally in HKMK's work. An illustration of this flapping is given in Figure 15(a), which shows a time history of the difference between wall pressure signals recorded at two points located $30^{\circ}$ to either side of the downstream $\theta=0$ direction, $p\left(-30^{\circ}\right)-p\left(30^{\circ}\right)$, at a depth of $z=-0.09 D$ below the cavity opening. The pressure difference is presented in non-dimensional $C_{p}$ form, as a function of non-dimensional time $t / T_{c}$ with the free stream convection time across the cavity, $T_{c}$, equal to $1.1 \times 10^{-3} \mathrm{~s}$ in this study. The pressure difference was low-pass filtered by a first order filter with a cut-off frequency of

(a)

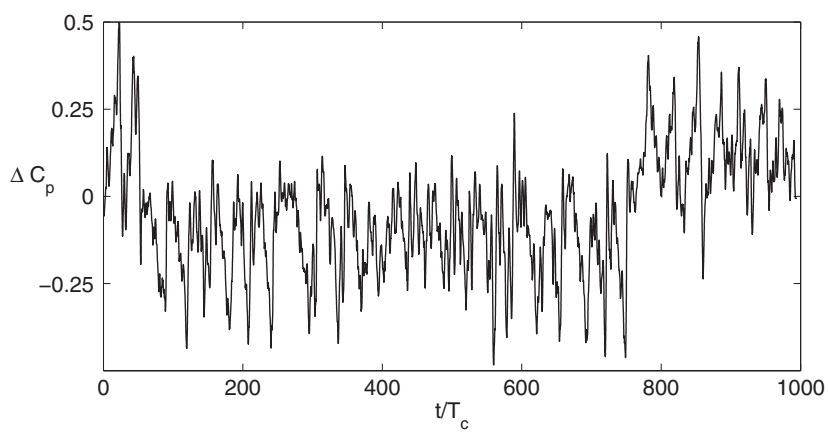

(b)

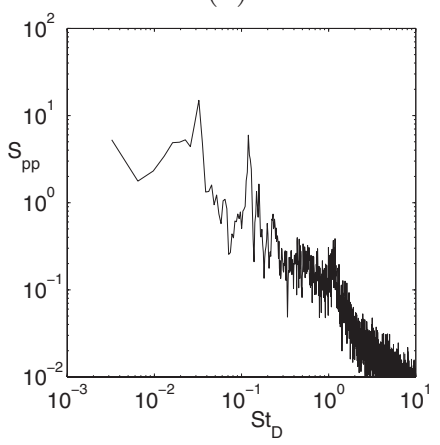

FIG. 15. (a) Difference $\Delta C_{p}(t)$ between time-dependent pressure coefficients at points located $30^{\circ}$ to either side of the downstream $\theta=0$ direction, below the cavity opening, and (b) power spectral density $S_{p p}$ of one of the two pressure signals, scaled by the local mean squared fluctuating pressure, represented as a function of the diameter-based Strouhal number $S t_{D}$ $=f D / U_{\infty}=f T_{c}$. Cavity of depth ratio $\kappa=0.32$. 
(a)

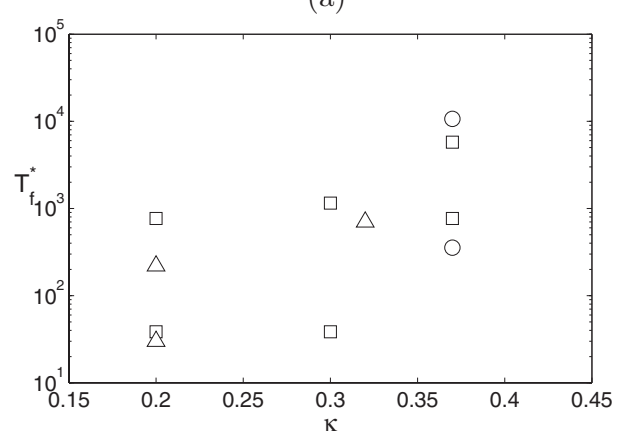

(b)

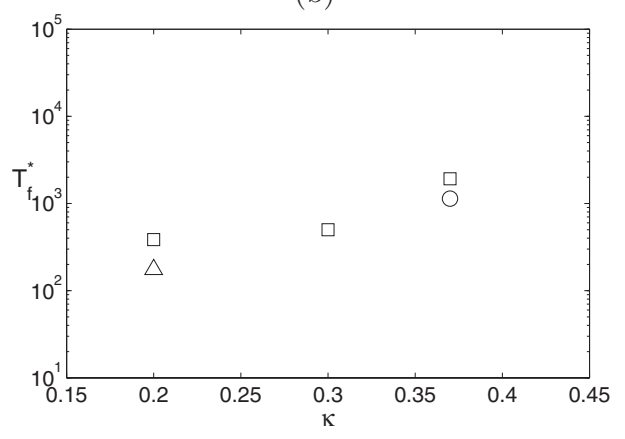

FIG. 16. Flapping time normalized by the convection time $T_{f}^{*}=T_{f} / T_{c}$ as a function of depth ratio $\kappa$. (a) Extreme values: $(\square) \mathrm{HKMK},{ }^{15}$ (०) Dybenko, ${ }^{17}$ and $(\triangle)$ present computations. (b) Estimated average values.

$500 \mathrm{~Hz}$, or equivalently a diameter-based Strouhal number of $S t_{D}=0.56$, in order to highlight the low frequency content. A total of 1000 convection times are shown for the $\kappa=0.32$ configuration.

At least three distinct time scales are visible in the pressure difference. First, there are high frequency pressure fluctuations $S t_{D} \geq 1$ beyond the cutoff frequency of the low-pass filter, attributable to turbulent velocity fluctuations along the cavity walls. Similar high-frequency noise was detected by Dybenko, ${ }^{17}$ but less so by HKMK perhaps due to bandwidth limitations of their static pressure transducers. This high frequency content is better illustrated in Figure 15(b), which shows the power spectral density of one of the two pressure traces scaled by the local mean squared pressure and plotted as a function of Strouhal number $S t_{D}$. The PSD of the other pressure trace, not shown here, is very close to the one displayed. Second, the pressure difference features notable periodic oscillations at a period of around 30 fly-over times. During these oscillations, the flow inside the cavity appears not to switch fully from one side to the other, since the pressure difference does not change sign, but instead wobbles between a roughly symmetrical state $\left(\Delta C_{p}=0\right)$ and an asymmetrical one $\left(\Delta C_{p} \neq 0\right)$. In the spectral domain, the period of these oscillations corresponds to a peak at a diameter-based Strouhal number of 0.03 , clearly visible as a wide peak in Figure 15(b). There is also a sharp peak visible in the PSD at a Strouhal number of $S t_{D}=0.12$, corresponding to a periodicity of $8 T_{c}$ in the time plots. Despite the high degree of emergence of these two peaks, their origin is unclear. Finally, over the entire length of the recorded pressure difference, one large scale flap is observed. At the beginning of the time history, the pressure difference is positive, indicating that the large cavity recirculation is skewed to the right. At $t / T_{c}=80$, the pressure difference changes sign to become on average negative, pointing to the recirculation being skewed to the left. The pressure difference switches back at $t / T_{c}=760$. The period of almost 700 fly-over times observed for this single switch is compared to experimental data from HKMK ${ }^{15}$ and Dybenko ${ }^{17}$ in Figures 16(a) and 16(b). Minimum and maximum flapping times estimated from the static pressure signals illustrated in the aforementioned publications, non-dimensionalized by the respective fly-over times, are displayed in Figure 16(a), while Figure 16(b) provides a rough estimation of the average values from their data as well as from the computations. It should be noted that the average values issued from the computations are averages over a small number of flaps, and therefore cannot be considered as converged. Despite the wide spread of observed switching periods for a given cavity depth found in the different studies, non-dimensional flapping times $T_{f}^{*}=T_{f} / T_{c}$ exhibit similar trends, with both extreme and average flapping times increasing with $\kappa$. The values of $T_{f}^{*}$ obtained at $\kappa=0.2$ and $\kappa=0.32$ lie within the range of experimental results.

The flapping flow inside the cavity is highlighted in Figure 17, which shows views of the norm of the instantaneous velocity at representative times before and after the flap at $t / T_{c}=760$, in the $z / H$ $=-0.1$ plane just below the cavity opening. The velocity fields are clearly skewed antisymmetrically between the two views, and the level of asymmetry is considerable. 
(a)

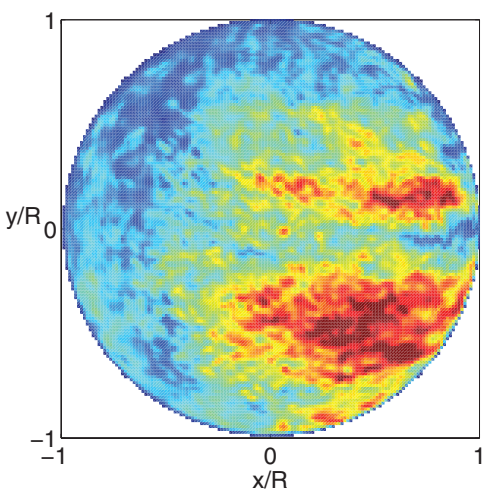

(b)

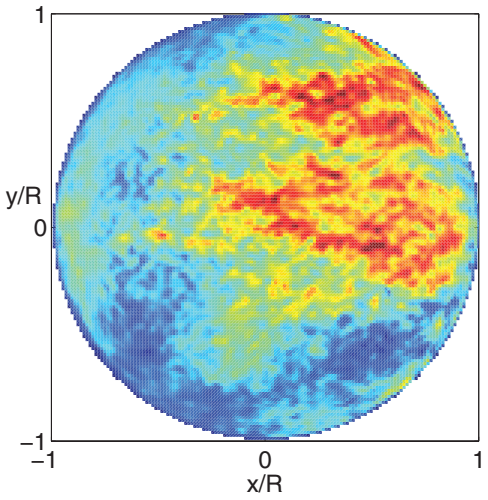

FIG. 17. Instantaneous flow norm, $\sqrt{u^{2}+v^{2}+w^{2}}$, non-dimensionalized by the free stream flow velocity, in the $z / H=-0.1$ plane, at times (a) $t / T_{c}=300$ and (b) at $t / T_{c}=900$. Colour scale between 0 and $0.5 \times U_{\infty}$.

Figure 18 shows the static pressure coefficient recorded on the cavity wall and floor for a cavity of depth ratio $\kappa=0.32$. The pressure distribution is roughly symmetrical, and bears a qualitative resemblence to the distribution found for a cavity of depth ratio $\kappa=1$, illustrated in Figure 4. The symmetry is not perfect, as it would be if the flapping occurred at random between two antisymmetrical flow states and averaging were performed over a sufficiently long period. Given the exceedingly low frequency of the flapping, the duration of the computational averaging is clearly not sufficiently long to achieve a converged long-term average value, which is probably the main reason for the asymmetry found in the computational mean results. It is nevertheless interesting to note that in HKMK's experiments, the measured static pressure was also almost but not quite symmetrical for all depths in the range $0.2 \leq \kappa<0.4$. The present pressure field for $\kappa=0.32$ is in good qualitative agreement with HKMK's data measured for $\kappa=0.37$, not shown here for the sake of brevity. The static pressure can almost be described as a mix between that of the symmetrical $\kappa=1$ cavity and that of the most strongly asymmetrical $\kappa=0.5$ cavity. The zone of maximum $C_{p}$ on the downstream cavity wall is centred around $\theta=10^{\circ}$ just off free stream flow direction, and is both larger and less intense than that of the $\kappa=0.5$ case shown in Figure 11, which is consistent with the long-term mean flow being the average of two antisymmetric flow states similar to that found for $\kappa=0.5$. Isopleths on the rest of the wall show strong remnants of the two intense negative zones present in the cavity of depth $\kappa=1$, although for the present cavity their positions are again not quite symmetrical. On

(a)

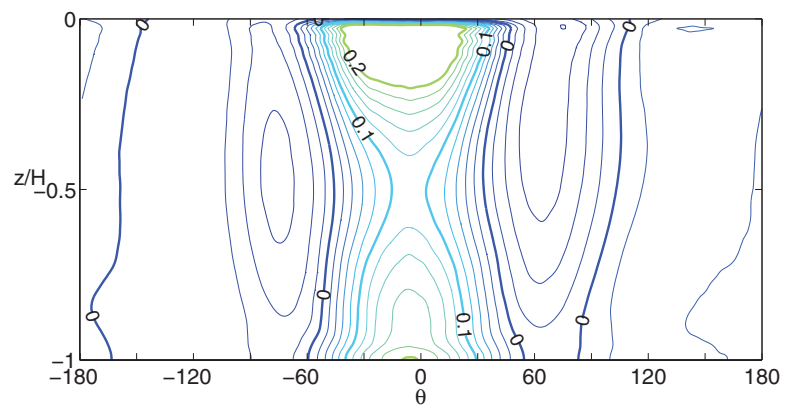

(b)

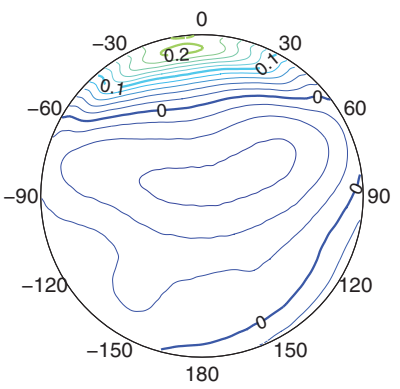

FIG. 18. Computed wall pressure (a) on the cavity wall and (b) floor for a cavity of depth ratio $\kappa=0.32$. Note that wall pressure representation is non-isometric, an isometric view can be found in Figure $7(\mathrm{~g})$. 


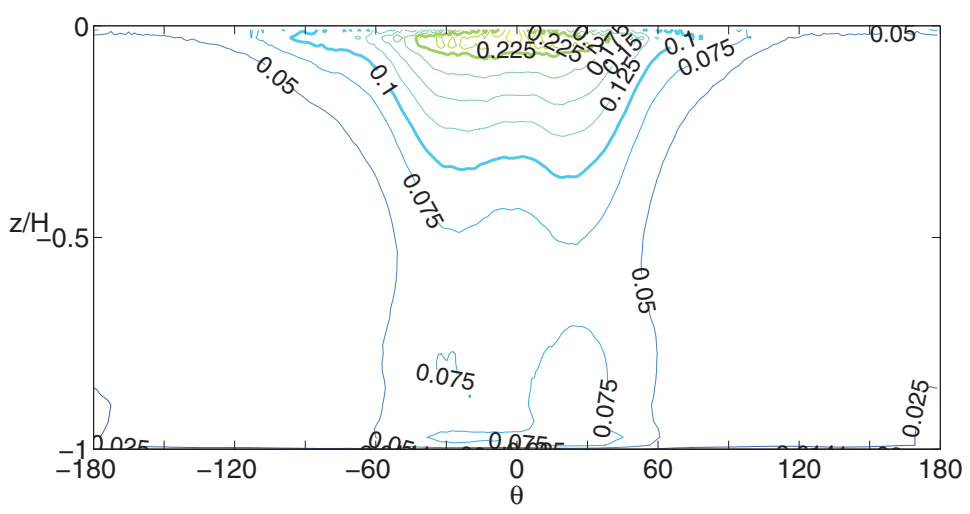

FIG. 19. Computed pressure fluctuations on the cavity wall for a cavity of depth $\kappa=0.32$, represented as non-dimensional $C_{p_{r m s}}$.

the cavity floor, a horseshoe-like zone of negative $C_{p}$ is again found, reminiscent of the $\kappa=1$ case, but substantially less curved and also skewed a little to one side. Also of note is a slight tongue of negative $C_{p}$ heading out from the centre of the cavity floor and pointing towards $\theta=135^{\circ}$, which corresponds to the direction of the large negative zone previously seen on the cavity floor of the $\kappa=0.5$ configuration. The progressive change in shape from the $\kappa=0.5$ to the $\kappa=0.32$ case is highlighted by the representation of static wall pressure for all the values of $\kappa$ studied in this work, in Figures $7(\mathrm{e})-7(\mathrm{~g})$.

Pressure fluctuations on the cavity's round wall, recorded for the same depth of $\kappa=0.32$, are shown in Figure 19. The levels of pressure fluctuations are very high, reaching values of $20 \%$ of the free stream dynamic pressure for $-30^{\circ} \leq \theta \leq 30^{\circ}$ and $z / H>-0.1$. Experimental data regarding pressure fluctuations in round cavities were gathered by Dybenko, ${ }^{17}$ for cavities of depth ratios $\kappa$ $=0.2,0.47$, and 0.7. The levels in the computations are considerably higher than those found by Dybenko, which do not exceed $C_{p_{r m s}}=0.12$ for a depth ratio of $\kappa=0.2$, and reach 0.08 for a depth ratio of $\kappa=0.47$. This is probably in part due to the very large thickness of the boundary layer in his experiment, which was performed with $\delta / D=0.72$. In the computations, the ratio of boundary layer thickness to cavity diameter is $\delta / D=0.15$, and thus it is expected that the shear layer impact on the downstream wall be more violent, both in terms of static $C_{p}$ and fluctuating $C_{p_{r m s}}$. Nevertheless, the high rms values in the computation are also in part due to the flow flapping, since the long-term average wall pressure and the shorter-term pressure averaged over the flapping time scale $T_{f}$ do not coincide. This point can be better understood by looking again at the wall pressure difference in Figure 15. For the two pressure locations, the long-term average pressure would be zero, while the short-term average, taken, for example, between $t / T_{c}=200$ and $t / T_{c}=600$, is -0.12 . Flow flapping is also responsible for the two distinct lobes of higher fluctuations, located $25^{\circ}$ to either side of the $\theta=0^{\circ}$ direction and extending from $z / H=0$ halfway down the cavity to $z / H=-0.5$, and which correspond to the two impact zones of the shear layer on the downstream cavity wall. At any given time, the shear layer preferentially impacts one side of the cavity, but with each flap this side is switched, leading to the symmetrical lobes in the long term time averaged pressure fluctuations.

Given the unusual nature of the flapping mean flow, rms velocity fluctuations are expected to be high throughout the cavity. This point is examined by representing mean and rms values of the three velocity components in the streamwise $y=0$ and cross stream $x=0$ planes, in Figures 20 and 21, respectively, for the $\kappa=0.32$ computation. Mean flow in the streamwise plane shows first an intense recirculation, with upstream-heading flow along the cavity floor reaching $-60 \mathrm{~m} \mathrm{~s}^{-1}$, or two-thirds of the free stream velocity $U_{\infty}$. The slight asymmetry of the mean flow is visible in the $V$ component, which, while weaker than in the highly asymmetrical $\kappa=0.5$ case, still reaches a maximum in the $y=0$ plane of $5 \%$ of $U_{\infty}$. In the same plane, rms values of the cross stream $v^{\prime}$ velocity component are strikingly high over the second half of the cavity, reaching more than $15 \%$ of $U_{\infty}$ over a large 
(a)

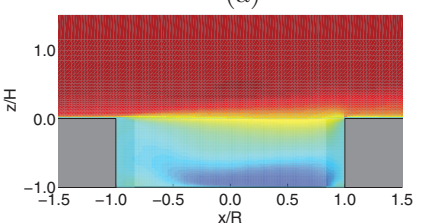

(d)

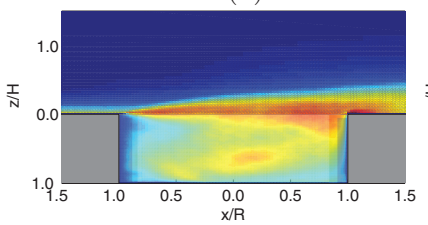

(b)

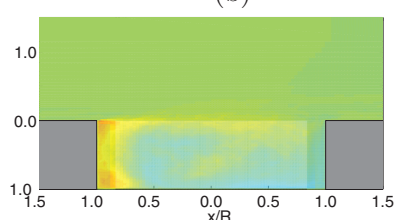

(e)

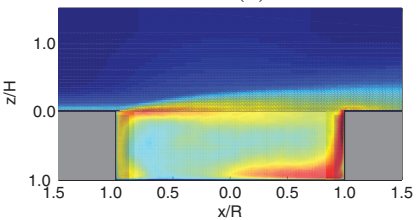

(c)

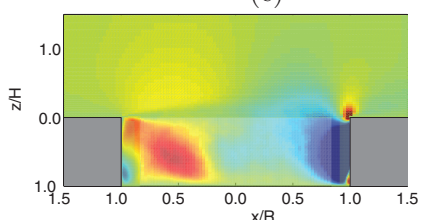

(f)

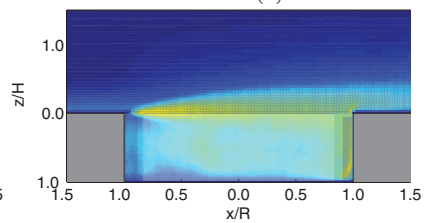

FIG. 20. Computed mean and rms values of (a, d) the streamwise $u,(\mathrm{~b}, \mathrm{e})$ the cross stream $v$ and $(\mathrm{c}, \mathrm{f})$ the vertical $w$ velocity fields in the $y=0$ plane, for a cavity of depth $\kappa=0.3$. Colour scales: [ -60100$] \mathrm{m} \mathrm{s}^{-1}$ for $U$, [-10 10] for $V$ and $W$, $\left[\begin{array}{ll}0 & 0.25\end{array}\right] \times U_{\infty}$ for rms components.

area close to the downstream bottom corner. Such large values of fluctuating velocity presumably reflect the large scale swings in short time mean flow observable from the previously described static pressure time histories. Vertical fluctuations in the shear layer are substantially less intense than those obtained for $\kappa=0.5$, reaching a maximum of $10 \%$ of free stream velocity. However, inside the cavity, $w_{r m s}^{\prime}$ reaches a high and almost uniform level of $6 \%$ of $U_{\infty}$. In the cross stream $x=0$ plane, the shear layer is no longer warped as was the case in the $\kappa=0.5$ computation, but flat, almost symmetrical, and thicker than in the $\kappa=0.5$ case. This increased thickness, as well as observed symmetry of the mean shear layer, may be explained by the long term averaging of two antisymmetrical states similar to those shown in Figures 14(d)-14(f) for the switching regime. There is also no distinct rotation around the $x$ axis, as shown by the plots of $V$ and $W$, which are almost antisymmetrical and symmetrical, respectively. Over the entire cavity width, substantial rms levels are observed, with an average for $u_{r m s}^{\prime}$ of around $10 \%$, and for $v_{r m s}^{\prime}$ and $w_{r m s}^{\prime}$, around $6 \%$ to $7 \%$ of the free stream velocity. Of note is the particularly high cross stream $v$ fluctuations visible near the cavity floor, where levels are as high as in the shear layer.

(a)

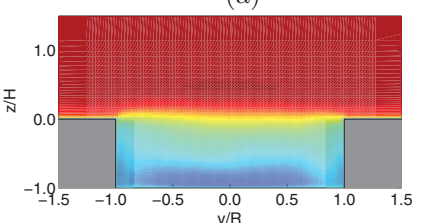

(d)

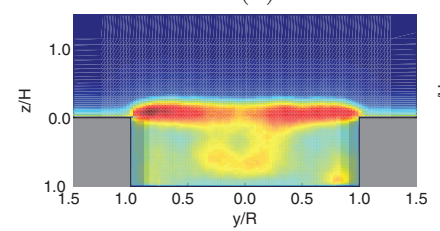

(b)

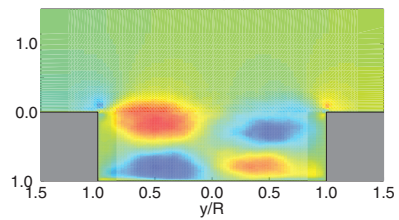

(e)

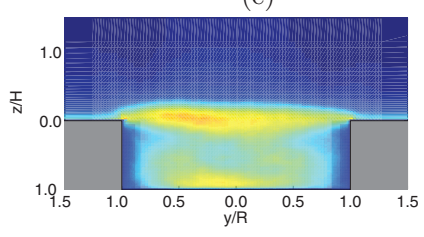

(c)

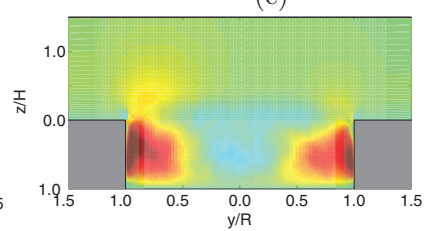

(f)

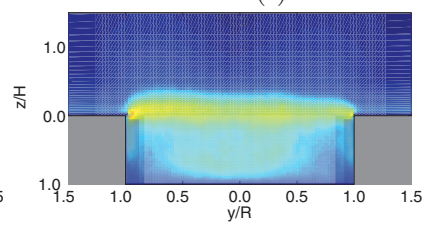

FIG. 21. Computed mean and rms values of (a, d) the streamwise $u$, (b, e) the cross stream $v$ and (c, f) the vertical $w$ velocity fields in the $x=0$ plane, for a cavity of depth $\kappa=0.3$. See Figure 20 for colour scales. 
(a)

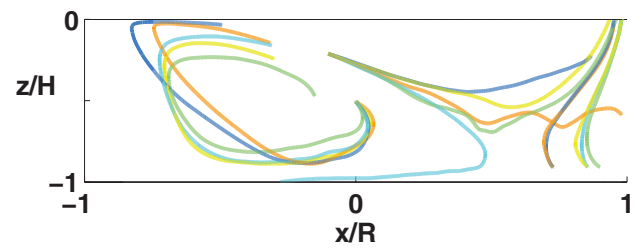

(b)

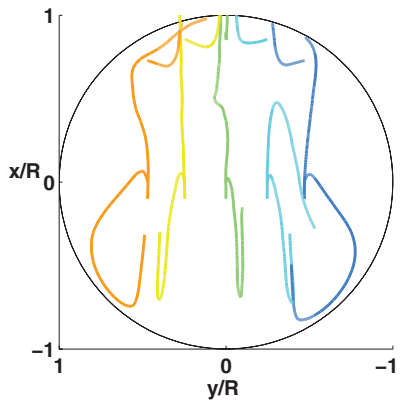

FIG. 22. (a) Side view and (b) top view of streamlines computed from the mean flow inside a cavity of depth ratio $\kappa=0.1$. Side view is not to scale, for enhanced readability. Streamline seed locations at $(r / R=0.42, z / H=-0.05)$, separated by an angle of $\pi / 16$.

\section{Depth ratio $0.1 \leq \kappa<0.2$}

According to HKMK, very shallow cavities of depth ratio $\kappa<0.2$ exhibit once again a stable symmetric mean flow. A computation performed for a depth ratio of $\kappa=0.1$ is described here to confirm this analysis. In Figures 22(a) and 22(b), respectively, streamlines computed inside the cavity are shown, viewed from the side and from the top. The side view shows two counter-rotating vortex structures, typical of the transitional flows found ${ }^{35,36}$ in rectangular cavities with ratios of length to depth roughly between 6 and 10. Both the large upstream vortex, centred around $(x / R=-0.3, z / H$ $=-0.5)$, and the smaller downstream vortex around $(x / R=0.9, z / H=-0.75)$, exhibit surprisingly little dependence on the cross-stream $y$ coordinate, as shown by the high degree of similarity between the different streamlines. The top view of the streamlines shows that the transversal mean velocity field $V$ is smaller than for the deeper cavities. This is confirmed by the examination of the static pressure field on the cavity floor, shown in Figure 7(j), where $C_{p}$ contours are seen to be parallel to the $y$ direction over most of the cavity floor.

\section{Large scale forces resulting from cavity flow}

As observed by a number of researchers, ${ }^{13-15}$ the drag induced by the presence of a cylindrical cavity is a non-monotonic function of the depth ratio $\kappa$, contrary to trends reported for rectangular cavities. ${ }^{14}$ A compendium of available drag results for round cavities can be found in Dybenko. ${ }^{17}$ Gaudet and Winter ${ }^{14}$ noted that drag is an almost cyclical function of depth, while Dybenko and Savory ${ }^{16}$ proposed that the state of the shear layer, and in particular the presence or not of large-scale organized vortices, might be responsible for this cyclical variation.

Rather than represent physical drag as a function of $\kappa$, the cavity's effect can be described as a time-dependent additional drag coefficient $\Delta C_{d}(t)=\left(\tau_{c}(t)-\tau_{w}\right) /\left(0.5 \rho U_{\infty}^{2}\right)$ where $\tau_{c}(t)$ is the time-varying effective wall shear stress induced by the cavity and $\tau_{w}$ the mean wall shear stress in the incoming boundary layer. This additional drag coefficient can then be normalized by the mean skin friction coefficient $C_{f}$ in the upstream boundary layer, yielding $\Delta C_{d} / C_{f}=\tau_{c} / \tau_{w}-1$. Gaudet and Winter ${ }^{14}$ showed that variations in the mean value $\Delta C_{d} / C_{f}$ can be well modeled by a power law built on the diameter-based friction velocity Reynolds number $u_{\tau} D / \nu$, where $u_{\tau}=\sqrt{\tau_{w} / \rho}$,

$$
\Delta C_{d} / C_{f}=A\left(u_{\tau} D / v\right)^{B},
$$

where $A$ is a function of both Mach number $\mathrm{M}$ and depth ratio $\kappa$, while $B$ is a function only of Mach number. Accordingly, in this work, $A$ is examined as a function of $\kappa$, for a fixed Mach number of $\mathrm{M}$ $=0.25$. The value of $B$ taken here is 0.32 , following values proposed by Gaudet and Winter ${ }^{14}$ and Pallister. ${ }^{37}$ 


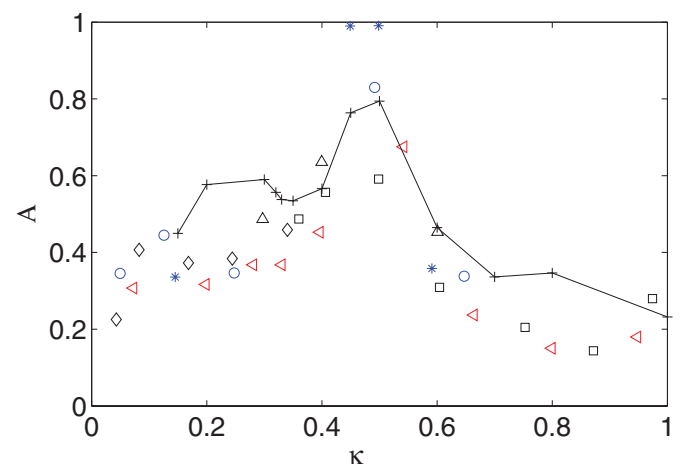

FIG. 23. Drag parameter $A$ from Eq. (1) as a function of cavity depth ratio $\kappa$. Symbols: experimental data from Tillman ${ }^{38}$ ( $\circ$ and $\left.{ }^{*}\right)$, Wieghardt ${ }^{13}\left(\square\right.$ and $\triangle$ ), Friesing ${ }^{1}(\triangleleft)$, and Gaudet and Winter $^{14}(\diamond)$. Solid line: computational results.

It can be noted that this approach is not favourable to absolute comparisons between numerical computations and experimental data, because of the unreasonable computational cost associated with accurately simulating a turbulent boundary layer at a realistic Reynolds number-of the order of 10000 for the momentum thickness based Reynolds number $\mathrm{Re}_{\theta}$ for the cavity flows in Marsden et al. ${ }^{12}$ - over a large spatial extent. With the relatively coarse grid at the wall, it is expected that the wall shear stress in the upstream boundary layer be substantially lower than that of the experiment, and indeed the friction velocity in the incoming boundary layer is smaller by a factor of four. This point is discussed in greater detail in the Appendix.

In order to compare the present results with available experimental data, $A$ is computed after normalizing $\Delta C_{d}$ with the experimental value ${ }^{12}$ of the boundary layer skin friction coefficient $C_{f_{e x p}}$ rather than the value obtained from the computations. The friction Reynolds number is also based on the experimental value of the friction velocity $u_{\tau_{e x p}}$. With this correction, a reasonable qualitative match is obtained, and presented in Figure 23. As is observed in the experimental data from a number of research campaigns, a maximum value of $A$ and hence of drag is obtained for $\kappa=0.5$, and drag falls off to either side of this value. The falloff to either side of $\kappa=0.5$ is weaker in the computational data than in the experimental results. At all depths, the supplemental cavity drag coefficient is almost entirely due to pressure forces, with wall shear forces inside the cavity remaining below $2 \%$ of pressure-induced drag.

Given the dominant pressure contribution to the total cavity drag, the location inside the cavity responsible for the increased drag can be examined from the wall pressure. This is done in Figure 24, which shows the angular distribution of the cavity drag coefficient due to pressure, for

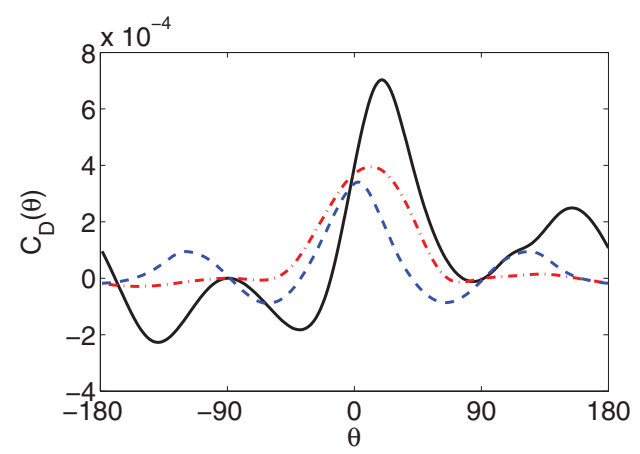

FIG. 24. Angular distribution of cavity pressure drag coefficient, $C_{D}(\theta)$, for cavity depths of (a) $\kappa=0.5(-)$; (b) $\kappa=0.8$ $(---)$; (c) $\kappa=0.2(---)$. 
cavity depths of $\kappa=0.2,0.5$, and 0.8 . This angular quantity $C_{D}(\theta)$ is defined here by

$$
C_{D}=1 / A \iint C_{p}(\theta, z) R \mathrm{~d} \theta \mathrm{d} z=\int C_{D}(\theta) \mathrm{d} \theta,
$$

where $A=\pi R^{2}$ is the cavity mouth area and $\theta$ is expressed in degrees. Both the shallow, $\kappa=0.2$, and deep, $\kappa=0.8$, cavities exhibit approximately symmetrical distributions of drag, with a maximum contribution around the downstream $\theta=0$ direction where the shear layer impacts the cavity wall. As expected, the peak for the cavity of depth ratio $\kappa=0.2$ is wider than that of the deeper cavity, due to the previously described flow switching and its associated anti-symmetrical wall pressure fields. The peak in the case of the cavity of depth ratio $\kappa=0.5$ is substantially stronger than for the two other cavities. It is off center, its maximum being located around $\theta=30^{\circ}$, as could be inferred from the wall pressure distribution shown in Figure 11. This zone corresponds to the downstream impact location of the warped shear layer on the cavity wall, as previously described in Sec. III B 2. There are also some notable local contributions to drag on the cavity upstream side, $C_{D}(\theta)>0$ for $\theta>90^{\circ}$ and $C_{D}(\theta)<0$ for $\theta<-90^{\circ}$, but the integral over these two zones is very small, and thus the additional drag observed for the cavity of depth ratio $\kappa=0.5$ is directly attributable to the shear layer's stronger impact on the downstream wall.

Given the asymmetrical flow encountered for depth ratios $\kappa$ between 0.4 and 0.6 , the pressure field inside the cavity also generates a transversal mean force, which can loosely be referred to as lift. This lift force is, however, weak in comparison to the drag force, since it reaches a maximum of only $8 \%$ of the latter, for a depth ratio of $\kappa=0.5$. Finally, in the flapping flow regime, unsteady forces are generated by the flow jumping between its two skewed states, but again these forces remain small compared to the mean drag. It can be noted that unsteady lift and drag forces due to flow flapping will occur at very low frequencies. As an example of this, the lift to drag ratio recorded for the cavity of $\kappa=0.2$ reaches values of $\pm 10 \%$, with a period of the order of 200 free stream convection times $T_{c}$ across the cavity. For the configuration studied in this work, with a cavity diameter of 0.1 $\mathrm{m}$, depth $0.02 \mathrm{~m}$, and a free stream Mach number of $\mathrm{M}=0.25$, this corresponds to a fluctuating lift force of around $0.1 \mathrm{~N}$ at a frequency of roughly $4 \mathrm{~Hz}$.

\section{CONCLUSIONS}

The different flow regimes found in shallow cylindrical cavities with depths smaller than their diameter have been investigated by compressible large eddy simulations. Depending on the cavity depth, mean flow is observed to be either symmetrical, for both very shallow and deep cavities, or asymmetrical, for intermediate cavity depths. In the asymmetrical case, mean flow can either be bistable, or exhibit very low frequency self-switching between two antisymmetrical states. The different flow regimes proposed in the literature for shallow cylindrical cavities, as well as the depth intervals for which they are encountered, are thus successfully reproduced in the computations. Numerical results compare favourably to the data available in the literature, namely, static pressure distributions, and non-monotonic variation of drag with cavity depth. Mean velocity fields and fluctuation maps are discussed for each regime, and time signals are described for self-switching configurations. Time scales associated with the self-switching are shown to vary strongly with cavity depth.

\section{ACKNOWLEDGMENTS}

This work was granted access to the HPC resources of the IDRIS under the allocation 2012020204 made by GENCI (Grand Equipement National de Calcul Intensif).

\section{APPENDIX: INCOMING BOUNDARY LAYER PARAMETERS AND EXCITATION}

The reference computation in this work, namely, the cavity of depth ratio $\kappa=1$, was designed to match experimental parameters found in the study of Marsden et al. ${ }^{19}$ The cavity in that study had a diameter and a depth of $D=H=0.1 \mathrm{~m}$, and was placed in a flat plate subject to grazing flow. 
Measurements were performed at various free stream Mach numbers, including that chosen for this work, of $M=0.25$. The boundary layer developing over the flat plate had a boundary layer thickness of $\delta_{99}=1.6 \times 10^{-2} \mathrm{~m}$, and a shape factor of $H=1.35$. Turbulence levels in the free stream were observed to be inferior to $1 \%$ of the free stream velocity. Profiles of measured mean and rms velocity are given in Marsden et al. ${ }^{12,19}$ The friction velocity estimated from the boundary layer profile is $u_{\tau}$ $=2.35 \mathrm{~m} \mathrm{~s}^{-1}$.

If the computation of the boundary layer were the aim of this work, a standard criterion of placing the first wall-normal grid point at a height of $z^{+} \simeq 1$ would be required, and the resulting wall-normal physical spacing would be $z=\eta / u_{\tau} \simeq 6.4 \times 10^{-6} \mathrm{~m}$, to be compared to $\delta_{99}=1.6$ $\times 10^{-2} \mathrm{~m}$. Moreover, a relatively large zone of the flat plate, of the order of a few cavity diameters in both the streamwise $x$ and the cross stream $y$ directions, is included in the computational domain, which would lead to entirely unreasonable numbers of points if criteria such as $x^{+} \simeq 20$ and $y^{+}$ $\simeq 10$ were applied for the construction of the grid.

Instead, a highly disturbed boundary layer with mean and rms characteristics roughly matching those observed in the experiment is reproduced in the present simulations. The method is developed with both highly relaxed grid requirements and aeroacoustic computations in mind.

The mean velocity profile prescribed at the inlet boundary condition is determined by fitting experimental data with a single equation model including a wake term. ${ }^{29}$ Incidentally this procedure provides an estimate of the friction velocity $u_{\tau}$ in the experiments, which cannot be obtained directly from the experimental profile due to the distance at which the first experimental velocity point is taken. This estimated value of $u_{\tau}$ is used elsewhere in this work.

A little downstream of the inlet zone, 25 grid points downstream in this work, deterministic perturbations are added inside the boundary layer over a height of $\delta_{\text {exc }}$, in the form of time derivatives of the velocity field in conservative form. These fluctuations are defined as modes each determined by a frequency $f_{m}$, intensities $I_{u_{m}}, I_{v_{m}}, I_{w_{m}}$, and a transversal wavelength, $\lambda_{y_{m}}$, as follows:

$$
\left\{\begin{array}{l}
\left.\frac{\partial \rho u}{\partial t}\right|_{m}=I_{u_{m}} \rho_{\infty} U_{\infty}\left(2 \pi f_{m}\right) \sin \left(2 \pi f_{m} t\right) \sin \left(\frac{2 \pi z}{\delta_{\text {exc }}}\right) \sin \left(\frac{2 \pi y}{\lambda_{y_{m}}}+\phi_{m}\right) \\
\left.\frac{\partial \rho v}{\partial t}\right|_{m}=I_{v_{m}} \rho_{\infty} U_{\infty}\left(2 \pi f_{m}\right) \sin \left(2 \pi f_{m} t\right) \sin \left(\frac{2 \pi z}{\delta_{\text {exc }}}\right) \sin \left(\frac{2 \pi y}{\lambda_{y_{m}}}+\phi_{m}\right) \\
\left.\frac{\partial \rho w}{\partial t}\right|_{m}=-I_{w_{m}} \rho_{\infty} U_{\infty}\left(2 \pi f_{m}\right) \sin \left(2 \pi f_{m} t\right) \sin \left(\frac{2 \pi z}{\delta_{\text {exc }}}\right) \sin \left(\frac{2 \pi y}{\lambda_{y_{m}}}+\phi_{m}\right)
\end{array}\right.
$$

with parameters involved given in Table II. Both transversal wavelengths $\lambda_{y m}$ and excitation thickness $\delta_{\text {exc }}$ are normalized by the boundary layer thickness, while frequencies $f_{m}$ are normalized with the time scale $\delta / U_{\infty}$. The values provided in Table II were empirically chosen for the present study.

The spectral contents of the perturbed boundary layer generated as previously described is now illustrated. Power spectral densities $\phi_{x x}$ of the fluctuating streamwise velocity are presented for five streamwise locations, at distances of $3 \delta, 6 \delta, 9 \delta$, and $10.5 \delta$ downstream of the excitation zone and at a distance of $1.6 \delta$ upstream of the excitation zone, in Figure 25. The spectra are computed as a function of streamwise wavenumber $k_{x}$ thanks to Taylor's frozen turbulence hypothesis, which leads to the following relation between time frequency and wavenumber: $k_{x}=2 \pi f / U_{c}$ where $U_{c}$ is the local convection speed, here taken as the mean flow velocity at the location of interest. The spectra are plotted as a function of $k_{x}$ multiplied by the boundary layer thickness $\delta$, and scaled such that $\int_{0}^{\infty} \phi_{x x}\left(k_{x}\right) d k_{x}=u_{r m s}^{2}$. The excitation frequencies $k_{x} \delta=2.2$ and $k_{x} \delta=3.15$, as well as a number

TABLE II. Numerical parameters for 2-mode boundary layer excitation procedure.

\begin{tabular}{lcccccc}
\hline \hline & $f_{m} \delta / U_{\infty}$ & $\lambda_{y_{m}} / \delta$ & $I_{u_{m}}$ & $I_{v_{m}}$ & $I_{w_{m}}$ & $\delta_{\text {exc }} / \delta$ \\
\hline $\mathrm{m}=1$ & 0.35 & 0.65 & 0.1 & 0.04 & 0.04 & 0.2 \\
$\mathrm{~m}=2$ & 0.50 & 0.40 & 0.1 & 0.04 & 0.04 & 0.2 \\
\hline \hline
\end{tabular}




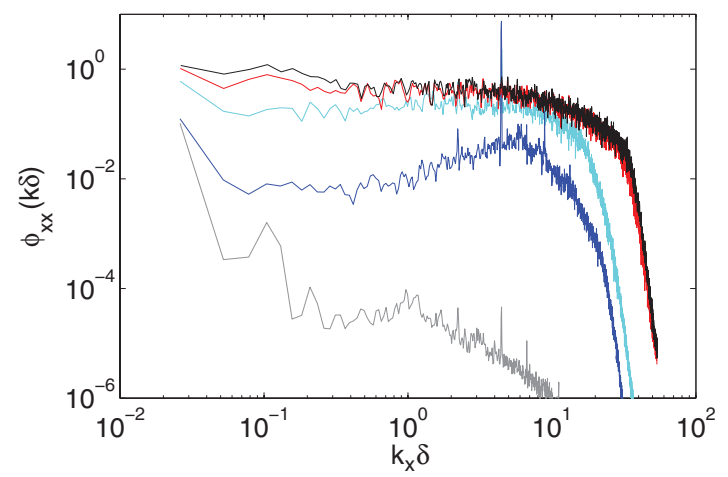

FIG. 25. Velocity spectra upstream and downstream of the boundary layer excitation zone, computed at distances of (-) $x / \delta$ $=-1.6 ;(-$, blue $) x / \delta=3 ;(-$, green) $x / \delta=6 ;(-$, red $) x / \delta=9 ;$ and $(-) x / \delta=10.5$ relative to the excitation zone, and at a height of $z^{+}=50$.

of their harmonics, are clearly visible at the first downstream location, where they emerge from background noise by around two orders of magnitude. These discrete frequencies are still visible at the next measurement location, six boundary-layer thicknesses downstream of the excitation region, but their amplitude is diminished, and the background fluctuations have increased by a factor of five to ten. At the third location, there is no trace of the excitation frequencies, and indeed the PSD is relatively free of discrete frequencies altogether. It is almost indistinguishable from the PSD measured at the fourth location, indicating that the boundary layer is no longer evolving rapidly in the streamwise direction. The spatial filtering used in the LES methodology generates a grid-based cut-off wavenumber above which fluctuations are strongly damped. With the eleven point filtering scheme of Bogey and Bailly, ${ }^{21}$ this cut-off can be considered to be around $k \Delta x=\pi / 2$, yielding values of $k_{x} \delta$ at cut-off of $15,20,36$, and 36 for the first four locations, respectively. The shift in $k_{x} \delta$ is due to the progressive streamwise mesh refinement approaching the cavity. These cut-off values of $k_{x} \delta$ are clearly visible on each curve, and materialized by a sharp decline in the spectra.

Figure 26 illustrates the resulting boundary layer profiles, both mean and fluctuating, in the present study, in outer units. The peak in $u_{r m s}$ is located at an altitude of 50 wall units, which is, as could be expected with the coarse wall-normal grid spacing, much higher than the height of around 15 wall units at which the peak is observed in high Reynolds number boundary layers. ${ }^{39}$ This overestimated height is not deemed of primary importance in this work focusing on the flow dynamics inside the cavities.

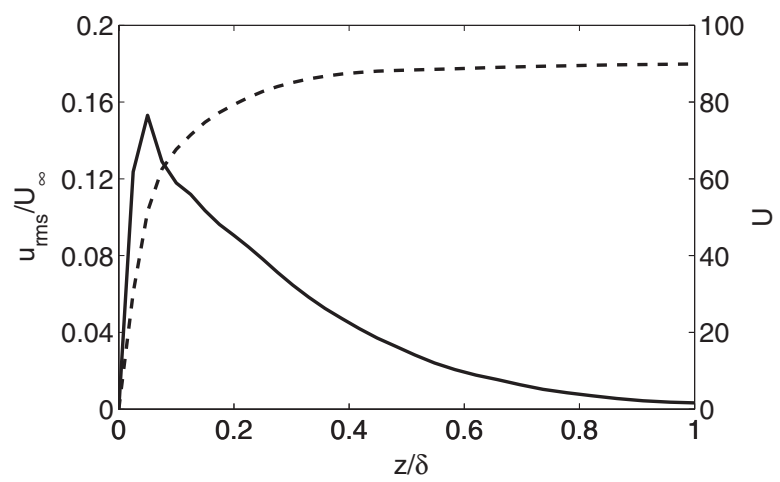

FIG. 26. Profiles of mean velocity (-- ) and rms fluctuations (-) generated in the boundary layer upstream of the cavity at $x / R=-1.2$. 
In summary, the strategy adopted in this work allowed the generation of a boundary layer of the desired thickness with high levels of velocity fluctuations of $15 \%$, as seen in Figure 26, without generating noticeable levels of spurious noise, at a reasonable computational cost. No claims are made here that this boundary layer excitation procedure would provide satisfactory results in different circumstances.

${ }^{1}$ H. Friesing, "Measurement of the drag associated with recessed surfaces: cut-outs of rectangular and elliptical planform," Zentralbl Bakteriol Parasitenkd Infektionskr Hyg. 628 (1936) (RAE library translation 1614, 1971).

${ }^{2}$ N. H. Fletcher, "Stopped-pipe wind instruments: Acoustics of the panpipes," J. Acoust. Soc. Am. 117, 370-374 (2005).

${ }^{3}$ J. Coltman, "Jet drive mechanisms in edge tones and organ pipes," J. Acoust. Soc. Am. 60, 725-733 (1976).

${ }^{4}$ Y. Yang, D. Rockwell, K. Lai-Fook Cody, and M. Pollack, "Generation of tones due to flow past a deep cavity: Effect of streamwise length," Technical Report No. LM-06K055 2006.

${ }^{5}$ D. Rockwell and E. Naudascher, "Review-Self-sustaining oscillations of flow past cavities," ASME J. Fluids Eng. 100, 152-165 (1978)

${ }^{6}$ M. S. Howe, "The dissipation of sound at an edge," J. Sound Vib. 70, 407-411 (1980).

${ }^{7}$ J. C. Bruggeman, "Flow-induced pulsations in pipe systems," Ph.D dissertation (Eindhoven University of Technology, 1987).

${ }^{8}$ W. Jungowski, K. Botros, and W. Studzinski, “Cylindrical side-branch as tone generator," J. Sound Vib. 131, 265-285 (1989).

${ }^{9}$ S. Ziada and S. Shine, "Strouhal numbers of flow-excited acoustic resonance of closed side branches," J. Fluids Struct. 13, 127-142 (1999).

${ }^{10}$ S. A. Elder, "Self-excited depth-mode resonance for a wall-mounted cavity in turbulent flow," J. Acoust. Soc. Am. 64, 877-890 (1978)

${ }^{11}$ S. P. Parthasarathy, Y. I. Cho, and L. H. Back, "Sound generation by flow over relatively deep cylindrical cavities," J. Acoust. Soc. Am. 78, 1785-1795 (1985).

${ }^{12}$ O. Marsden, C. Bailly, C. Bogey, and E. Jondeau, "Investigation of flow features and acoustic radiation of a round cavity," J. Sound Vib. 331, 3521-3543 (2012).

${ }^{13}$ K. Wieghardt, "Increase of the turbulent frictional resistance caused by surface irregularities," MAP R \& T No. 103,1946 (translation of ZWB FB 15639 1942).

${ }^{14}$ L. Gaudet and K. G. Winter, "Measurements of the drag of some characteristic aircraft excrescences immersed in turbulent boundary layers," Technical Report No. TM AERO 1538 (Royal Aircraft Establishment, 1973).

${ }^{15}$ M. Hiwada, T. Kawamura, I. Mabuchi, and M. Kumada, "Some characteristics of flow pattern and heat transfer past a circular cylindrical cavity,” Bull. JSME 26, 1744-1752 (1983).

${ }^{16}$ J. Dybenko and E. Savory, "An experimental investigation of turbulent boundary layer flow over surface-mounted circular cavities,” J. Aerosp. Eng. 222, 109-125 (2008).

${ }^{17}$ J. Dybenko, "An experimental investigation of turbulent boundary surface-layer flow over surface-mounted circular cavities," Ph.D. dissertation (The University of Western Ontario, 2005).

${ }^{18}$ C. Haigermoser, F. Scarano, and M. Onorato, "Investigation of the flow in a circular cavity using stereo and tomographic particle image velocimetry," Exp. Fluids 46, 517-526 (2009).

${ }^{19}$ O. Marsden, E. Jondeau, P. Souchotte, C. Bailly, C. Bogey, and D. Juvé, "Investigation of flow features and acoustic radiation of a round cavity," AIAA Paper 2008-2851 2008.

${ }^{20} \mathrm{C}$. Bogey and C. Bailly, "Turbulence and energy budget in a self-preserving round jet: Direct evaluation using large-eddy simulation,” J. Fluid Mech. 627, 129-160 (2009).

${ }^{21}$ C. Bogey and C. Bailly, "A family of low dispersive and low dissipative explicit schemes for noise computations," J. Comput. Phys. 194, 194-214 (2004).

22 J. Berland, C. Bogey, O. Marsden, and C. Bailly, "High-order, low dispersive and low dissipative explicit schemes for multiple-scale and boundary problems," J. Comput. Phys. 224, 637-662 (2007).

${ }^{23}$ E. Garnier, N. Adams, P. Sagaut, E. Garnier, N. Adams, and P. Sagaut, "Explicit structural modeling," in Large Eddy Simulation for Compressible Flows, Scientific Computation (Springer, The Netherlands, 2009), pp. 95-118.

${ }^{24}$ C. Bogey and C. Bailly, "Large eddy simulations of transitional round jets: Influence of the Reynolds number on flow development and energy dissipation," Phys. Fluids 18, 1-14 (2006).

${ }^{25}$ C. Bogey and C. Bailly, "Computation of a high Reynolds number jet and its radiated noise using large eddy simulation based on explicit filtering," Comput. Fluids 35, 1344-1358 (2006).

${ }^{26}$ O. Marsden, C. Bogey, and C. Bailly, "Direct noise computation of the turbulent flow around a zero-incidence airfoil," AIAA J. 46, 874-883 (2008).

${ }^{27}$ C. K. W. Tam and F. Hu, "An optimized multidimensional interpolation scheme for computational aeroacoustics applications using overset grids," AIAA Paper 2004-2812 2004.

${ }^{28}$ D. Desvigne, O. Marsden, C. Bogey, and C. Bailly, "Development of non-centered wavenumber-based optimized interpolation schemes with amplification control for overlapping grids," SIAM J. Sci. Comput. 32, 2074-2098 (2010).

${ }^{29}$ S. E. Guarini, R. D. Moser, K. Shariff, and A. Wray, "Direct numerical simulation of a supersonic turbulent boundary layer at Mach 2.5," J. Fluid Mech. 414, 1-33 (2000).

${ }^{30}$ T. Cebeci and P. Bradshaw, Momentum Transfer in Boundary Layers (Hemisphere Pub. Corp., Washington, 1977).

${ }^{31}$ J. Dybenko and E. Savory, "An experimental investigation of turbulent boundary layer flow over surface-mounted circular cavities," CSME Forum (2006).

${ }^{32}$ A. C. Kistler and F. C. Tan, "Some properties of turbulent separated flows," Phys. Fluids 10(9), S165-S173 (1967), The Physics of Fluids Supplement: Boundary Layers and Turbulence. 
${ }^{33}$ V. O’Brien, "Closed streamlines associated with channel flow over a cavity," Phys. Fluids 15, 2089-2097 (1972).

${ }^{34}$ A. Roshko, "Some measurements of flow in a rectangular cutout," Technical Note 3488 (NACA, 1955).

${ }^{35}$ M. B. Tracy and E. B. Plentovich, "Characterization of cavity flow fields using pressure data obtained in the Langley $0.3-\mathrm{m}$ transonic cryogenic tunnel,” Technical Report No. 4436 (NASA Technical Memorandum, 1993).

${ }^{36}$ L. Chatellier, "Modélisation et contrôle actif des instabilités aroacoustiques en cavit sous coulement affleurant," Ph.D. dissertation (Université de Poitiers, 2002).

${ }^{37}$ K. C. Pallister, "Wind tunnel measurements of the transonic drag of excrescences immersed in a turbulent boundary layer," ARA. Report No. 371974.

${ }^{38}$ W. Tillman, "Additional measurements of the drag of surface irregularities in a turbulent boundary layer," NACA TM (1951).

${ }^{39}$ I. Marusic, B. J. McKeon, P. A. Monkewitz, H. M. Nagib, A. J. Smits, and K. R. Sreenivasan, "Wall-bounded turbulent flows at high Reynolds numbers: Recent advances and key issues,” Phys. Fluids 22, 065103 (2010). 